\title{
Pacific
}

Journal of

Mathematics

\section{PARAMETRIZATION OF HOLOMORPHIC SEGRE-PRESERVING MAPS}

\author{
R. BLAIR ANGLE
}




\title{
PARAMETRIZATION OF HOLOMORPHIC SEGRE-PRESERVING MAPS
}

\author{
R. BLAIR ANGLE
}

\begin{abstract}
We investigate holomorphic Segre-preserving maps sending the complexification $M$ of a generic real analytic submanifold $M \subseteq \mathbb{C}^{N}$ of finite type at some point $p$ into the complexification $\mathcal{M}^{\prime}$ of a generic real analytic submanifold $M^{\prime} \subseteq \mathbb{C}^{N^{\prime}}$ that is finitely nondegenerate at some point $p^{\prime}$. We prove that for a fixed $M$ and $M^{\prime}$, the germs at $(p, \bar{p})$ of Segre submersive holomorphic Segre-preserving maps sending $(M,(p, \bar{p}))$ into $\left(\mathcal{M}^{\prime},\left(p^{\prime}, \bar{p}^{\prime}\right)\right)$ rationally depend upon their $K$-jets at $(p, \bar{p})$, for some fixed $K$ depending only on $M$ and $M^{\prime}$, and these maps are uniquely determined by their $K$ jets. If, in addition, $M$ and $M^{\prime}$ are algebraic, we prove that any such map must be algebraic. It follows that the set of germs at $(p, \bar{p})$ of holomorphic Segre-preserving automorphisms of the complexification $M$ of a generic real analytic submanifold $M$ that is finitely nondegenerate and of finite type at $p$ is an algebraic complex Lie group. We explore the relationship between this automorphism group and the group of automorphisms of $M$ at $p$.
\end{abstract}

\section{Introduction}

Let $M \subseteq \mathbb{C}^{N}$ be a real analytic submanifold of codimension $d$, defined locally near $p \in M$ by the real-valued real analytic function $\rho(Z, \bar{Z})$. The complexification $M$ of $M$ is a holomorphic submanifold of $\mathbb{C}^{2 N}$ given locally for $(Z, \zeta) \in \mathbb{C}^{N} \times \mathbb{C}^{N}$ near $(p, \bar{p})$ by $\mathcal{M}=\{(Z, \zeta): \rho(Z, \zeta)=0\}$. Assume $M$ is generic (see Section 2), and let $M^{\prime} \subseteq \mathbb{C}^{N^{\prime}}$ be a generic real analytic submanifold of codimension $d^{\prime}$, let $p^{\prime} \in M^{\prime}$, and let $\mathcal{M}^{\prime}$ denote its complexification. Let $U \subseteq \mathbb{C}^{N}$ be an open neighborhood of $p$, and define ${ }^{*} U:=\{\bar{Z}: Z \in U\}$. Consider a holomorphic map $\mathscr{H}:\left(U \times{ }^{*} U,(p, \bar{p})\right) \rightarrow$ $\left(\mathbb{C}^{2 N^{\prime}},\left(p^{\prime}, \bar{p}^{\prime}\right)\right)$ of the form

$$
\mathscr{H}(Z, \zeta)=(H(Z), \widetilde{H}(\zeta)),
$$

where $H: U \rightarrow \mathbb{C}^{N^{\prime}}$ and $\widetilde{H}:{ }^{*} U \rightarrow \mathbb{C}^{N^{\prime}}$. Assume that $\mathscr{H}\left(\mathcal{M} \cap\left(U \times{ }^{*} U\right)\right) \subseteq \mathcal{M}^{\prime}$. These maps will be the chief object of study in this paper. We will call such a map

MSC2000: 32A10, 32H02, 32H12, 32V35, 32V40.

Keywords: holomorphic Segre-preserving map, complexification, real analytic submanifold, finite jet determination, algebraic submanifold, finitely nondegenerate, finite type. 
a holomorphic Segre-preserving map (HSPM) since it preserves Segre varieties in a sense that will be made precise in Section 2. With the notation $\bar{\varphi}(z):=\overline{\varphi(\bar{z})}$, we observe that if $\widetilde{H}=\bar{H}$, then $H$ is a holomorphic map defined on $U$ sending $(M \cap U, p)$ into $\left(M^{\prime}, p^{\prime}\right)$. Such maps have been extensively studied. However, HSPMs are relatively new; see [Zhang 2007; Angle 2008a; 2008b] for related recent work. Under certain restrictions, the collection of germs of HSPMs sending $(\mathcal{M},(p, \bar{p}))$ into $\left(\mathcal{M}^{\prime},\left(p^{\prime}, \bar{p}^{\prime}\right)\right)$ is, in a manner to be detailed in subsequent sections, "bigger" than the collection of germs of holomorphic mappings sending $(M, p)$ into $\left(M^{\prime}, p^{\prime}\right)$. We shall see several examples of this in Section 5.

For $p_{0} \in \mathbb{C}^{m}$, let $\mathscr{T}_{p_{0}}\left(\mathbb{C}^{m}\right)$ denote the holomorphic tangent space of $\mathbb{C}^{m}$ at $p_{0}$. Let $\mathscr{T}_{(p, \bar{p})}^{0}, \mathcal{M} \subseteq \mathscr{T}_{(p, \bar{p})}\left(\mathbb{C}^{2 N}\right)$ denote the set of all vectors of the form

$$
\sum_{j=1}^{N} a_{j} \frac{\partial}{\partial Z_{j}}+\sum_{j=1}^{N} b_{j} \frac{\partial}{\partial \zeta_{j}} \quad \text { such that } \sum_{j=1}^{N} a_{j} \frac{\partial}{\partial Z_{j}} \quad \text { and } \quad \sum_{j=1}^{N} b_{j} \frac{\partial}{\partial \bar{Z}_{j}}
$$

are tangent to $M$ at $p$. A vector of the form $\sum_{j=1}^{N} a_{j}\left(\partial / \partial Z_{j}\right)$ tangent to $M$ at $p$ is known as a holomorphic tangent vector, and a vector of the form $\sum_{j=1}^{N} b_{j}\left(\partial / \partial \bar{Z}_{j}\right)$ tangent to $M$ at $p$ is known as an antiholomorphic tangent vector. For any germ at $(p, \bar{p})$ of an HSPM $\mathscr{H}$ sending $(\mathcal{M},(p, \bar{p}))$ into $\left(\mathcal{M}^{\prime},\left(p^{\prime}, \bar{p}^{\prime}\right)\right)$, we have

$$
\mathscr{D}_{(p, \bar{p})} \mathscr{H}\left(\mathscr{T}_{(p, \bar{p})}^{0} \mathcal{M}\right) \subseteq \mathscr{T}_{\left(p^{\prime}, \bar{p}^{\prime}\right)}^{0} \mathcal{M}^{\prime},
$$

where we define

$$
\mathscr{D}_{(p, \bar{p})} \mathscr{H}: \mathscr{T}_{(p, \bar{p})}\left(\mathbb{C}^{2 N}\right) \rightarrow \mathscr{T}_{\left(p^{\prime}, \bar{p}^{\prime}\right)}\left(\mathbb{C}^{2 N^{\prime}}\right) \quad \text { by } \quad\left(\mathscr{D}_{(p, \bar{p})} \mathscr{H}(\mathscr{L})\right)(\varphi)=\mathscr{L}(\varphi \circ \mathscr{H})
$$

for any germ at $\left(p^{\prime}, \bar{p}^{\prime}\right)$ of a holomorphic function $\varphi: \mathbb{C}^{2 N^{\prime}} \rightarrow \mathbb{C}$. We say that $\mathscr{H}$ is Segre submersive at $(p, \bar{p})$ if

$$
\mathscr{D}_{(p, \bar{p})} \mathscr{H}\left(\mathscr{T}_{(p, \bar{p})}^{0} \mathcal{M}\right)=\mathscr{T}_{\left(p^{\prime}, \bar{p}^{\prime}\right)}^{0} \mathcal{M}^{\prime} .
$$

This definition is independent of choice of coordinates for $M$ and $M^{\prime}$.

Given $M$ and $M^{\prime}$ satisfying certain geometric conditions, our main result is Theorem 1.1, which states that the germs at $(p, \bar{p})$ of HSPMs that are Segre submersive at $(p, \bar{p})$ and send $(\mathcal{M},(p, \bar{p}))$ into $\left(\mathcal{M}^{\prime},\left(p^{\prime}, \bar{p}^{\prime}\right)\right)$ rationally depend upon their $K$-jets, for some fixed $K$ depending only on $M$ and $M^{\prime}$. This result was motivated by, and is a generalization of, results due to Baouendi, Ebenfelt, and Rothschild [1999a] and Baouendi, Rothschild, and Zaitsev [2001]. We also mention a recent paper of Lamel and Mir [2007] for related results. Before stating Theorem 1.1, we present some more notation. Let $J^{K}\left(\mathbb{C}^{N}, \mathbb{C}^{N^{\prime}}\right)_{\left(p, p^{\prime}\right)}$ denote the set of $K$-jets at $p$ of germs of holomorphic maps from $\left(\mathbb{C}^{N}, p\right)$ into $\left(\mathbb{C}^{N^{\prime}}, p^{\prime}\right)$. (In this paper, we assume that $J^{K}\left(\mathbb{C}^{N}, \mathbb{C}^{N^{\prime}}\right)_{\left(p, p^{\prime}\right)}$ includes only derivatives of positive 
order.) Let $j_{p}^{K}$ represent the corresponding $K$-jet map defined on the set of germs at $p$ of holomorphic mappings given by

$$
j_{p}^{K} \phi=\left(\frac{\partial^{|\alpha|} \phi}{\partial Z^{\alpha}}(p)\right)_{1 \leq|\alpha| \leq K} .
$$

Theorem 1.1. Let $M \subseteq \mathbb{C}^{N}$ be real analytic, generic, and of finite type at $p$. Let $M^{\prime} \subseteq \mathbb{C}^{N^{\prime}}$ be real analytic, generic, and finitely nondegenerate at $p^{\prime}$. Assume that $M$ is of codimension $d$, that $M^{\prime}$ is of codimension $d^{\prime}$, and that $N-d \geq N^{\prime}-d^{\prime}$. Then there exist positive integers

$$
K \geq 1 \quad \text { and } \quad 1 \leq r \leq\left(\begin{array}{c}
N-d \\
N^{\prime}-d^{\prime}
\end{array}\right)^{2}
$$

depending only on $M$ and $M^{\prime}$, and $\mathbb{C}^{N^{\prime}}$-valued holomorphic functions $\Phi_{1}, \ldots, \Phi_{r}$ defined on an open subset of $\mathbb{C}^{N} \times J^{K}\left(\mathbb{C}^{N}, \mathbb{C}^{N^{\prime}}\right)_{\left(p, p^{\prime}\right)} \times J^{K}\left(\mathbb{C}^{N}, \mathbb{C}^{N^{\prime}}\right)_{\left(\bar{p}, \bar{p}^{\prime}\right)}$ of the form

$$
\Phi_{l}(Z, \Lambda, \Gamma)=\sum_{\gamma} \frac{P_{\gamma}^{l}(\Lambda, \Gamma)}{Q_{1}^{l}(\Lambda)^{s_{\gamma}^{l}} Q_{2}^{l}(\Gamma)^{t_{\gamma}^{l}}}(Z-p)^{\gamma},
$$

where $s_{\gamma}^{l}$ and $t_{\gamma}^{l}$ are nonnegative integers, $P_{\gamma}^{l}$ are $\mathbb{C}^{N^{\prime}}$-valued polynomials, and $Q_{1}^{l}$ and $Q_{2}^{l}$ are $\mathbb{C}$-valued polynomials with real coefficients, such that the following holds. Let $\mathscr{H}(Z, \zeta)=(H(Z), \widetilde{H}(\zeta))$ be a germ at $(p, \bar{p})$ of an HSPM sending $(\mathcal{M},(p, \bar{p}))$ into $\left(\mathcal{M}^{\prime},\left(p^{\prime}, \bar{p}^{\prime}\right)\right)$ such that $\mathcal{H}$ is Segre submersive at $(p, \bar{p})$. Then there exists $l$, with $1 \leq l \leq r$, such that

$$
\begin{aligned}
H(Z) & =\Phi_{l}\left(Z, j_{p}^{K}(H), j_{\bar{p}}^{K}(\widetilde{H})\right), \\
\widetilde{H}(\zeta) & =\bar{\Phi}_{l}\left(\zeta, j_{\bar{p}}^{K}(\widetilde{H}), j_{p}^{K}(H)\right),
\end{aligned}
$$

for $(Z, \zeta)$ sufficiently close to $(p, \bar{p})$. Furthermore, for any

$$
\left(\Lambda_{0}, \Gamma_{0}\right) \in J^{K}\left(\mathbb{C}^{N}, \mathbb{C}^{N^{\prime}}\right)_{\left(p, p^{\prime}\right)} \times J^{K}\left(\mathbb{C}^{N}, \mathbb{C}^{N^{\prime}}\right)_{\left(\bar{p}, \bar{p}^{\prime}\right)}
$$

such that $Q_{1}^{l}\left(\Lambda_{0}\right) \neq 0$ and $Q_{2}^{l}\left(\Gamma_{0}\right) \neq 0$, the map $\Phi_{l}$ is holomorphic in a neighborhood of $\left(p, \Lambda_{0}, \Gamma_{0}\right)$.

The appearance of $\bar{\Phi}_{l}$ in (1-4) is interesting and will be instrumental in the proof of Corollary 1.4. See Section 2 for precise definitions of finite type and finite nondegeneracy. 


\section{Define}

$\operatorname{Aut}(M, p):=\left\{H:\left(\mathbb{C}^{N}, p\right) \rightarrow\left(\mathbb{C}^{N}, p\right) \mid H\right.$ is a germ at $p$ of a holomorphic map, $H$ is invertible at $p$, and $H(M) \subseteq M\}$, $\operatorname{Aut}_{\mathbb{C}}(\mathcal{M},(p, \bar{p})):=\left\{\mathscr{H}:\left(\mathbb{C}^{2 N},(p, \bar{p})\right) \rightarrow\left(\mathbb{C}^{2 N},(p, \bar{p})\right) \mid \mathscr{H}\right.$ is a germ at $(p, \bar{p})$ of an HSPM, $\mathscr{H}$ is invertible at $(p, \bar{p})$, and $\mathscr{H}(\mathcal{M}) \subseteq \mathcal{M}\}$.

We call $\operatorname{Aut}(M, p)$ the group of automorphisms of $M$ at $p$, and $\operatorname{Aut}_{\mathbb{C}}(M,(p, \bar{p}))$ the group of holomorphic Segre-preserving automorphisms of $\mathcal{M}$ at $(p, \bar{p})$. Let $J_{p}^{K}\left(\mathbb{C}^{N}\right):=J^{K}\left(\mathbb{C}^{N}, \mathbb{C}^{N}\right)_{(p, p)}$ be a simplification of notation, define $G_{p}^{K}\left(\mathbb{C}^{N}\right)$ to be the set of all elements of $J_{p}^{K}\left(\mathbb{C}^{N}\right)$ that correspond to invertible mappings at $p$, and define a jet map $\eta_{(p, \bar{p})}^{K}$ on the set of germs at $(p, \bar{p})$ of HSPMs such that for $\mathscr{H}=(H, \widetilde{H}), \eta_{(p, \bar{p})}^{K}(\mathscr{H}):=\left(j_{p}^{K} H, j_{\bar{p}}^{K} \widetilde{H}\right)$. Noting that $M=M^{\prime}$ implies that $r=1$ in Theorem 1.1, we have the following corollary.

Corollary 1.2. Let $M \subseteq \mathbb{C}^{N}$ be a generic real analytic submanifold of finite type at $p$ and finitely nondegenerate at $p$. Then there exists an integer $K$ depending only on $M$ such that $\eta_{(p, \bar{p})}^{K}$ restricted to $\operatorname{Aut}_{\mathbb{C}}(\mathcal{M},(p, \bar{p}))$ is a homeomorphism onto a closed, holomorphic algebraic submanifold (Lie group) of $G_{p}^{K}\left(\mathbb{C}^{N}\right) \times G_{\bar{p}}^{K}\left(\mathbb{C}^{N}\right)$.

Remark 1.3. One consequence of Corollary 1.2 is that $j_{p}^{K}$ restricted to $\operatorname{Aut}(M, p)$ is a homeomorphism onto a closed, real algebraic submanifold (Lie group) of $G_{p}^{K}\left(\mathbb{C}^{N}\right)$. This fact has already been proven in previous work. For $M$ a hypersurface, Baouendi, Ebenfelt, and Rothschild [1997] showed that $j_{p}^{K}(\operatorname{Aut}(M, p))$ is a closed, real analytic submanifold (Lie group) of $G_{p}^{K}\left(\mathbb{C}^{N}\right)$. However, they did not show that it is also real algebraic. In [1999a], they proved this fact for $M$ a submanifold of any codimension.

Since $j_{p}^{K}(\operatorname{Aut}(M, p))$ is a real algebraic submanifold, it is natural to consider its complexification as a holomorphic submanifold of $G_{p}^{K}\left(\mathbb{C}^{N}\right) \times G_{\bar{p}}^{K}\left(\mathbb{C}^{N}\right)$. We will denote this complexification $\mathbb{C}\left\{j_{p}^{K}(\operatorname{Aut}(M, p))\right\}$. What is the relationship between $\mathbb{C}\left\{j_{p}^{K}(\operatorname{Aut}(M, p))\right\}$ and $\eta_{(p, \bar{p})}^{K}\left(\operatorname{Aut}_{\mathbb{C}}(M,(p, \bar{p}))\right)$ ? The following corollary of Theorem 1.1 says that the former is always contained in the latter, and they are necessarily of the same dimension. In Section 5 we will give examples demonstrating both equality and strict containment.

Corollary 1.4. Let $M$ and $K$ be as in Corollary 1.2. Let $\mathscr{\Im} \subseteq G_{p}^{K}\left(\mathbb{C}^{N}\right) \times G_{\bar{p}}^{K}\left(\mathbb{C}^{N}\right)$ denote the connected component of $\mathbb{C}\left\{j_{p}^{K}(\operatorname{Aut}(M, p))\right\}$ containing $\left(\operatorname{Id}, \operatorname{Id}^{\prime}\right)$, where Id (respectively, $\left.\mathrm{Id}^{\prime}\right)$ is the point in $G_{p}^{K}\left(\mathbb{C}^{N}\right)$ (respectively, $G_{\bar{p}}^{K}\left(\mathbb{C}^{N}\right)$ ) corresponding to the identity map on $\mathbb{C}^{N}$. Let $\mathscr{C} \subseteq G_{p}^{K}\left(\mathbb{C}^{N}\right) \times G_{\bar{p}}^{K}\left(\mathbb{C}^{N}\right)$ denote the connected component of $\eta_{(p, \bar{p})}^{K}\left(\operatorname{Aut}_{\mathbb{C}}(\mathcal{M},(p, \bar{p}))\right)$ that contains $\left(\mathrm{Id}, \mathrm{Id}^{\prime}\right)$. Then

(i) $\mathbb{C}\left\{j_{p}^{K}(\operatorname{Aut}(M, p))\right\} \subseteq \eta_{(p, \bar{p})}^{K}\left(\operatorname{Aut}_{\mathbb{C}}(M,(p, \bar{p}))\right)$, 
(ii) $\mathscr{B}=\mathscr{C}$, and

(iii) $\eta_{(p, \bar{p})}^{K}\left(\operatorname{Aut}_{\mathbb{C}}(\mathcal{M},(p, \bar{p}))\right)$ and $\mathbb{C}\left\{j_{p}^{K}(\operatorname{Aut}(M, p))\right\}$ are made up of finitely many disjoint cosets of $\mathscr{B}$.

The following application of Theorem 1.1 states that Segre submersive HSPMs sending $(\mathcal{M},(p, \bar{p}))$ into $\left(\mathcal{M}^{\prime},\left(p^{\prime}, \bar{p}^{\prime}\right)\right)$ are uniquely determined by a finite number of derivatives.

Theorem 1.5. Let $M$ and $M^{\prime}$ be as defined in Theorem 1.1. Then there exists an integer $K$, depending only on $M$ and $M^{\prime}$, such that the following holds. Let $\mathscr{H}_{1}(Z, \zeta)=\left(H_{1}(Z), \widetilde{H}_{1}(\zeta)\right)$ and $\mathscr{H}_{2}(Z, \zeta)=\left(H_{2}(Z), \widetilde{H}_{2}(\zeta)\right)$ be germs at $(p, \bar{p})$ of HSPMs sending $(\mathcal{M},(p, \bar{p}))$ into $\left(\mathcal{M}^{\prime},\left(p^{\prime}, \bar{p}^{\prime}\right)\right)$ such that $\mathscr{H}_{1}$ and $\mathscr{H}_{2}$ are Segre submersive at $(p, \bar{p})$. If

$$
j_{p}^{K}\left(H_{1}\right)=j_{p}^{K}\left(H_{2}\right) \quad \text { and } \quad j_{\bar{p}}^{K}\left(\widetilde{H}_{1}\right)=j_{\bar{p}}^{K}\left(\widetilde{H}_{2}\right),
$$

then $\mathscr{H}_{1}(Z, \zeta) \equiv \mathscr{H}_{2}(Z, \zeta)$.

One of the strengths of Theorem 1.1 lies in the fact that the form of $\Phi_{l}$ leads to Corollaries 1.2 and 1.4. These maps, however, depend upon the jets of both $H$ and $\widetilde{H}$. In Theorem 1.6, we see that it is in fact possible to find functions that express $\mathscr{H}$ entirely in terms of the $L$-jets of $H$ (or of $\widetilde{H}$ ) for some $L$.

Theorem 1.6. Let $M$ and $M^{\prime}$ be as in Theorem 1.1. Then there exist positive integers $r$ and $L$, depending only on $M$ and $M^{\prime}$, and $\mathbb{C}^{2 N^{\prime}}$-valued holomorphic functions $\Phi_{1}^{1}, \ldots, \Phi_{r}^{1}$ defined on an open subset of $\mathbb{C}^{2 N} \times J^{L}\left(\mathbb{C}^{N}, \mathbb{C}^{N^{\prime}}\right)\left(p, p^{\prime}\right)$ and $\Phi_{1}^{2}, \ldots, \Phi_{r}^{2}$ defined on an open subset of $\mathbb{C}^{2 N} \times J^{L}\left(\mathbb{C}^{N}, \mathbb{C}^{N^{\prime}}\right)_{\left(\bar{p}, \bar{p}^{\prime}\right)}$ such that the following holds. Let $\mathscr{H}(Z, \zeta)=(H(Z), \widetilde{H}(\zeta))$ be a germ at $(p, \bar{p})$ of an HSPM sending $(\mathcal{M},(p, \bar{p}))$ into $\left(\mathcal{M}^{\prime},\left(p^{\prime}, \bar{p}^{\prime}\right)\right)$ such that $\mathscr{H}$ is Segre submersive at $(p, \bar{p})$. Then there exist $1 \leq l_{1}, l_{2} \leq r$ such that

$$
\begin{aligned}
& \mathscr{H}(Z, \zeta)=\Phi_{l_{1}}^{1}\left(Z, \zeta, j_{p}^{L}(H)\right), \\
& \mathscr{H}(Z, \zeta)=\Phi_{l_{2}}^{2}\left(Z, \zeta, j_{\bar{p}}^{L}(\widetilde{H})\right)
\end{aligned}
$$

for $(Z, \zeta)$ sufficiently close to $(p, \bar{p})$.

The following example shows that Theorem 1.6 does not necessarily hold if $M^{\prime}$ is finitely degenerate at $p^{\prime}$.

Example 1.7. Let $M=M^{\prime} \subseteq \mathbb{C}^{2}$ be given by $M=\left\{\operatorname{Im} w=|z|^{4}\right\}$ and its complexification by $\mathcal{M}=\left\{w-\tau=2 i z^{2} \chi^{2}\right\}$, where $(z, w)$ and $(\chi, \tau)$ are coordinates on $\mathbb{C}^{2}$. We note that $M$ is of finite type but finitely degenerate at 0 . Let $H(z, w)=(z, w)$. We can find two distinct maps $\widetilde{H}_{1}(\chi, \tau)$ and $\widetilde{H}_{2}(\chi, \tau)$ such that $\mathscr{H}_{1}=\left(H, \widetilde{H}_{1}\right)$ and $\mathscr{H}_{2}=\left(H, \widetilde{H}_{2}\right)$ both send $(\mathcal{M}, 0)$ into $\left(\mathcal{M}^{\prime}, 0\right)$ and are Segre submersive at 0 . Indeed, let $\widetilde{H}_{1}(\chi, \tau)=(\chi, \tau)$ and let $\widetilde{H}_{2}(\chi, \tau)=(-\chi, \tau)$. 
Finally, we present a result on algebraicity. Recall that a real analytic mapping is said to be real analytic algebraic if all of its components are real analytic algebraic, and a real analytic submanifold is said to be real algebraic if it can be given by real analytic algebraic defining functions; similarly a holomorphic map is said to be holomorphic algebraic if all of its components are holomorphic algebraic, and a holomorphic submanifold is said to be holomorphic algebraic if it can be given by holomorphic algebraic defining functions.

Theorem 1.8. Let $M$ and $M^{\prime}$ be as in Theorem 1.1, and assume that $M$ and $M^{\prime}$ are real algebraic. Then any germ at $\left(p^{\prime}, \bar{p}^{\prime}\right)$ of an HSPM sending $(\mu,(p, \bar{p}))$ into $\left(\mathcal{M}^{\prime},\left(p^{\prime}, \bar{p}^{\prime}\right)\right)$ that is Segre submersive at $(p, \bar{p})$ is holomorphic algebraic.

The layout of this paper is as follows. In Section 2, we present some additional background material. Section 3 contains the reformulations and proofs of three of the main results as given in Section 1, while Section 4 is dedicated to proving the main results of Section 1. Section 5 consists of several examples of HSPMs and automorphism groups. In particular, examples demonstrating both equality and nonequality of $\mathbb{C}\left\{j_{p}^{K}(\operatorname{Aut}(M, p))\right\}$ and $\eta_{(p, \bar{p})}^{K}\left(\operatorname{Aut}_{\mathbb{C}}(M,(p, \bar{p}))\right)$ are provided. See [Angle 2008b] for additional examples.

\section{Additional background}

Let $M \subseteq \mathbb{C}^{N}$ be a real analytic submanifold of codimension $d$. Recall that this means that given any $p \in M$, there exists a real vector-valued real analytic function $\rho=\left(\rho_{1}, \ldots, \rho_{d}\right)$ defined in a neighborhood of $p$, satisfying $d \rho_{1} \wedge \cdots \wedge d \rho_{d} \neq 0$ at $p$, such that $M$ is given locally near $p$ by the vanishing of $\rho$. We refer to $\rho$ as the local defining function for $M$ near $p$. If, in addition, near any $p \in M$, there exists a local defining function $\rho$ satisfying the stronger condition $\partial_{Z} \rho_{1} \wedge \cdots \wedge \partial_{Z} \rho_{d} \neq 0$ at $p$, then we say that $M$ is generic. If $M$ is generic, it can be shown (see for example [Baouendi et al. 1999b]) that there exists a holomorphic change of coordinates $Z=(z, w) \in \mathbb{C}^{N-d} \times \mathbb{C}^{d}$ vanishing at $p$ and an open neighborhood $\Omega$ of 0 such that in these coordinates $M$ is locally given by $\{(z, w) \in \Omega: w=Q(z, \bar{z}, \bar{w})\}$, where $Q(z, \chi, \tau)=\left(Q^{1}(z, \chi, \tau), \ldots, Q^{d}(z, \chi, \tau)\right)$ is a $\mathbb{C}^{d}$-valued holomorphic function defined near 0 in $\mathbb{C}^{N-d} \times \mathbb{C}^{N-d} \times \mathbb{C}^{d}$ and satisfying $Q(0, \chi, \tau) \equiv Q(z, 0, \tau) \equiv \tau$. Such coordinates are called normal coordinates.

A vector field of the form $\sum_{j=1}^{N} a_{j}(Z, \bar{Z})\left(\partial / \partial \bar{Z}_{j}\right)$ tangent to $M$ near $p$, where the $a_{j}$ are smooth functions on $M$, is called a $C R$ vector field. We say that $M$ is of finite type at $p$ (in the sense of [Kohn 1972] and [Bloom and Graham 1977]) if the CR vector fields, their complex conjugates, and all repeated commutators of these vector fields span the complexified tangent space of $M$ at $p$. Letting $\left(\rho_{j}\right)_{Z}:=\left(\partial \rho_{j} / \partial Z_{1}, \ldots, \partial \rho_{j} / \partial Z_{N}\right)$ and $L^{\alpha}:=L_{1}^{\alpha_{1}} \cdots L_{m}^{\alpha_{m}}$, where $\alpha=\left(\alpha_{1}, \ldots, \alpha_{m}\right)$ and $L_{1}, \ldots, L_{m}$ is a basis for the CR vector fields of $M$ near $p$, we say that $M$ is 
finitely nondegenerate at $p$ if there exists a nonnegative integer $K$ such that

$$
\operatorname{span}\left\{L^{\alpha}\left(\rho_{j}\right)_{Z}(p):|\alpha| \leq K, 1 \leq j \leq d\right\}=\mathbb{C}^{N} .
$$

We say that $M$ is $k$-nondegenerate at $p$ if $k$ is the smallest $K$ for which (2-1) holds. It is not difficult to show that if $M$ is given in normal coordinates by $w=$ $Q(z, \bar{z}, \bar{w})$, then $M$ is $k$-nondegenerate at 0 if and only if the matrix whose rows are $\left(Q_{z_{l} \chi^{\alpha}}^{j}(0,0,0)\right)_{1 \leq l \leq N-d}$ for $|\alpha| \leq K$ and $1 \leq j \leq d$ has rank $N-d$ for $K \geq k$ and rank less than $N-d$ for $K<k$.

Let $M \subseteq \mathbb{C}^{N}$ be the generic real analytic submanifold $\{Z \in \Omega: \rho(Z, \bar{Z})=0\}$, where $\Omega$ is a nonempty open subset of $\mathbb{C}^{N}, \rho(Z, \zeta)$ is holomorphic on $\Omega \times{ }^{*} \Omega$, and $\partial_{Z} \rho(Z, \zeta) \neq 0$ for all $(Z, \zeta) \in \Omega \times{ }^{*} \Omega$. The complexification of $M$ is then given by $\mathcal{M}=\{(Z, \zeta) \in \Omega \times * \Omega: \rho(Z, \zeta)=0\}$. Given any $(Z, \zeta) \in \Omega \times * \Omega$, we define the Segre varieties of $M$ by

$$
\Sigma_{Z}:=\left\{\zeta \in{ }^{*} \Omega: \rho(Z, \zeta)=0\right\} \quad \text { and } \quad \widehat{\Sigma}_{\zeta}:=\{Z \in \Omega: \rho(Z, \zeta)=0\} .
$$

Segre varieties are named for the Italian geometer Beniamino Segre who introduced them [1931]. We note here that $\mathcal{M}$ is sometimes referred to as the Segre family associated with $M$; see [Chern 1975; Faran 1980], for example.

Now let $M^{\prime} \subseteq \mathbb{C}^{n+1}$ be the generic real analytic submanifold given by $M^{\prime}=$ $\left\{\left(Z^{\prime} \in \Omega^{\prime}: \rho^{\prime}\left(Z^{\prime}, \bar{Z}^{\prime}\right)=0\right\}\right.$, where $\Omega^{\prime}$ is a nonempty open subset of $\mathbb{C}^{n+1}, \rho^{\prime}\left(Z^{\prime}, \zeta^{\prime}\right)$ is holomorphic on $\Omega^{\prime} \times{ }^{*} \Omega^{\prime}$, and $\partial_{Z^{\prime}} \rho^{\prime}\left(Z^{\prime}, \zeta^{\prime}\right) \neq 0$ for all $\left(Z^{\prime}, \zeta^{\prime}\right) \in \Omega^{\prime} \times{ }^{*} \Omega^{\prime}$. Denote the complexification of $M^{\prime}$ by $\mathcal{M}^{\prime}$ and its Segre varieties by $\Sigma_{Z^{\prime}}^{\prime}$ and $\widehat{\Sigma}_{\zeta^{\prime}}^{\prime}$. Let $p \in M$ and $p^{\prime} \in M^{\prime}$, and let $\psi: \Omega \times{ }^{*} \Omega \rightarrow \mathbb{C}^{2 n+2}$ be a holomorphic map sending $(M,(p, \bar{p}))$ into $\left(\mathcal{M}^{\prime},\left(p^{\prime}, \bar{p}^{\prime}\right)\right)$. Furthermore, we will assume that for any $(Z, \zeta) \in \mathcal{M}$, there exists $\left(Z^{\prime}, \zeta^{\prime}\right) \in \mathcal{M}^{\prime}$ such that

$$
\begin{gathered}
\psi\left(\{Z\} \times \Sigma_{Z}\right) \subseteq\left\{Z^{\prime}\right\} \times \Sigma_{Z^{\prime}}^{\prime}, \\
\psi\left(\widehat{\Sigma}_{\zeta} \times\{\zeta\}\right) \subseteq \widehat{\Sigma}_{\zeta^{\prime}}^{\prime} \times\left\{\zeta^{\prime}\right\} .
\end{gathered}
$$

Proposition 2.1. The map $\psi$, when restricted to $\mathcal{M}$, is an HSPM of the form (1-1).

Faran [1980] proved this for hypersurfaces, but it also holds in higher codimension.

Proof. We show it is true in a neighborhood of $(Z, \zeta)=\left(Z_{0}, \zeta_{0}\right) \in M$. Write $\psi(Z, \zeta)=\left(\phi_{1}(Z, \zeta), \phi_{2}(Z, \zeta)\right)$, where $\phi_{1}$ and $\phi_{2}$ are $\mathbb{C}^{N^{\prime}}$-valued holomorphic functions, and write $\zeta_{0}=\left(\zeta_{0}^{\prime}, \zeta_{0}^{\prime \prime}\right) \in \mathbb{C}^{N-d} \times \mathbb{C}^{d}$. As $M$ is generic, the implicit function theorem implies that (after a possible rearrangement of coordinates) there exists a $\mathbb{C}^{d}$-valued holomorphic function $\theta$, satisfying $\theta\left(Z_{0}, \zeta_{0}^{\prime}\right)=\zeta_{0}^{\prime \prime}$, such that $\left(Z, \zeta_{0}^{\prime}, \theta\left(Z, \zeta_{0}^{\prime}\right)\right) \in \mathcal{M}$ for any $Z$ sufficiently close to $Z_{0}$. For any $Z$ near $Z_{0}$, define $H(Z):=\phi_{1}\left(Z, \zeta_{0}^{\prime}, \theta\left(Z, \zeta_{0}^{\prime}\right)\right)$. We claim that $H(Z)=\phi_{1}(Z, \zeta)$ on $M$. This is because (2-2) implies that $\phi_{1}\left(p_{0}, \zeta\right)$ is constant for all $\zeta \in \Sigma_{p_{0}}$ for any $p_{0}$. A similar argument applies to $\phi_{2}$. 


\section{Reformulations}

In this section, we will assume unless said otherwise that $M \subseteq \mathbb{C}^{m+d}$ and $M^{\prime} \subseteq \mathbb{C}^{n+e}$ are real analytic generic submanifolds of codimensions $d$ and $e$, respectively. We will additionally assume that there are open neighborhoods $0 \in U \subseteq \mathbb{C}^{m+d}$ and $0 \in U^{\prime} \subseteq \mathbb{C}^{n+e}$ such that $M$ is given by

$$
M=\{(z, w) \in U: w=Q(z, \bar{z}, \bar{w})\},
$$

where $Z=(z, w)$ are normal coordinates, and $M^{\prime}$ is given by

$$
M^{\prime}=\left\{\left(z^{\prime}, w^{\prime}\right) \in U^{\prime}: w^{\prime}=Q^{\prime}\left(z^{\prime}, \bar{z}^{\prime}, \bar{w}^{\prime}\right)\right\},
$$

where $Z^{\prime}=\left(z^{\prime}, w^{\prime}\right)$ are normal coordinates. We also assume that $\rho(z, w, \chi, \tau):=$ $w-Q(z, \chi, \tau)$ is holomorphic on $U \times{ }^{*} U$ and $\partial_{Z} \rho(z, w, \chi, \tau)$ is nonvanishing on $U \times{ }^{*} U$. Similarly, we assume that $\rho^{\prime}\left(z^{\prime}, w^{\prime}, \chi^{\prime}, \tau^{\prime}\right):=w^{\prime}-Q^{\prime}\left(z^{\prime}, \chi^{\prime}, \tau^{\prime}\right)$ is holomorphic on $U^{\prime} \times{ }^{*} U^{\prime}$ and $\partial_{Z^{\prime}} \rho^{\prime}\left(z^{\prime}, w^{\prime}, \chi^{\prime}, \tau^{\prime}\right)$ is nonvanishing on $U^{\prime} \times{ }^{*} U^{\prime}$. So the complexifications $\mathcal{M}$ and $\mathcal{M}^{\prime}$ of $M$ and $M^{\prime}$ are respectively given by

$$
M=\left\{(z, w, \chi, \tau) \in U \times{ }^{*} U: w=Q(z, \chi, \tau)\right\}
$$

and

$$
\mathcal{M}^{\prime}=\left\{\left(z^{\prime}, w^{\prime}, \chi^{\prime}, \tau^{\prime}\right) \in U^{\prime} \times{ }^{*} U^{\prime}: w^{\prime}=Q^{\prime}\left(z^{\prime}, \chi^{\prime}, \tau^{\prime}\right)\right\},
$$

where $\zeta=(\chi, \tau) \in \mathbb{C}^{m} \times \mathbb{C}^{d}$ and $\zeta^{\prime}=\left(\chi^{\prime}, \tau^{\prime}\right) \in \mathbb{C}^{n} \times \mathbb{C}^{e}$. Unless otherwise specified, we will assume any (germ of an) HSPM $\mathscr{H}$ sends $(\mathcal{M}, 0)$ into $\left(\mathcal{M}^{\prime}, 0\right)$ and is given in the form

$$
\mathscr{H}(Z, \zeta)=(H(Z), \widetilde{H}(\zeta))=(f(Z), g(Z), \tilde{f}(\zeta), \tilde{g}(\zeta)),
$$

where $f=\left(f^{1}, \ldots, f^{n}\right)$ and $\tilde{f}=\left(\tilde{f}^{1}, \ldots, \tilde{f}^{n}\right)$ are $\mathbb{C}^{n}$-valued holomorphic functions, $g=\left(g^{1}, \ldots, g^{e}\right)$ and $\tilde{g}=\left(\tilde{g}^{1}, \ldots, \tilde{g}^{e}\right)$ are $\mathbb{C}^{e}$-valued holomorphic functions, and we write $z=\left(z_{1}, \ldots, z_{m}\right), w=\left(w_{1}, \ldots, w_{d}\right), z^{\prime}=\left(z_{1}^{\prime}, \ldots, z_{n}^{\prime}\right)$, and $w^{\prime}=\left(w_{1}^{\prime}, \ldots, w_{e}^{\prime}\right)$, and similarly for $\chi, \tau, \chi^{\prime}$, and $\tau^{\prime}$.

Reformulation of Theorem 1.1. We begin with a technical definition.

Definition 3.1. Let $M \subseteq \mathbb{C}^{m+d}$ be of codimension $d$ and $M^{\prime} \subseteq \mathbb{C}^{n+e}$ be of codimension $e$, and assume $m \geq n$. Let $\mathscr{H}$ be an HSPM. Let $\mu=\left(\mu_{1}, \ldots, \mu_{n}\right)$ for some $1 \leq \mu_{1}<\cdots<\mu_{n} \leq m$ and $v=\left(v_{1}, \ldots, v_{n}\right)$ for some $1 \leq v_{1}<\cdots<v_{n} \leq m$, and assume that

$$
\operatorname{det}\left(\frac{\partial f^{k}}{\partial z_{\mu l}}(0)\right)_{1 \leq k, l \leq n} \neq 0 \quad \text { and } \operatorname{det}\left(\frac{\partial \tilde{f}^{k}}{\partial \chi_{\nu_{l}}}(0)\right)_{1 \leq k, l \leq n} \neq 0 .
$$

Then we say that the map $\mathscr{H}$ satisfies condition $D_{\mu \nu}$. 
Any given $\mathscr{H}$ may satisfy condition $D_{\mu \nu}$ for several different $\mu$ and $\nu$, as the following example illustrates.

Example 3.2. Let $M \subseteq \mathbb{C}^{4}$ and $M^{\prime} \subseteq \mathbb{C}^{3}$ be given by

$$
\begin{aligned}
M & =\left\{\operatorname{Im} w=\left|z_{1}\right|^{2}+2 \operatorname{Re}\left(z_{3} \bar{z}_{1}-z_{3} \bar{z}_{2}\right)-\left|z_{2}\right|^{2}\right\}, \\
M^{\prime} & =\left\{\operatorname{Im} w^{\prime}=\left|z_{1}^{\prime}\right|^{2}+\left|z_{2}^{\prime}\right|^{2}\right\} .
\end{aligned}
$$

Note that $M$ is of finite type at 0 , and $M^{\prime}$ is finitely nondegenerate at 0 . Let $\mathscr{H}$ be given by $\mathscr{H}(z, w, \chi, \tau)=\left(z_{1}+z_{3}, z_{1}-z_{2}, w, \chi_{1}-\chi_{2}, \chi_{2}+\chi_{3}, \tau\right)$. Then $\mathscr{H}$ satisfies condition $D_{\mu \nu}$ for any permissible $\mu$ and $\nu$. That is, $\mu$ can be any one of $(1,2)$, $(1,3)$, or $(2,3)$, as can $v$.

Our main theorem, from which Theorem 1.1 follows, is Theorem 3.3. Before we present it, we introduce some notation. Given an HSPM $\mathscr{H}$, we can write

$$
j_{0}^{K} H=\left(\left(f_{z l}^{j}(0)\right)_{1 \leq l \leq m, 1 \leq j \leq n},\left(j_{0}^{K}\right)^{\prime} H\right),
$$

where $\left(j_{0}^{K}\right)^{\prime} H$ represents the remaining derivatives of $H$ at 0 . Given any $\Lambda$ in $J_{0}^{K}\left(\mathbb{C}^{m+d}, \mathbb{C}^{n+e}\right)_{(0,0)}$, we will then write

$$
\Lambda=\left(\left(\Lambda^{j, l}\right)_{1 \leq l \leq m, 1 \leq j \leq n}, \Lambda^{\prime}\right),
$$

where $\left(j_{0}^{K} H\right)^{j, l}$ is exactly $f_{z l}^{j}(0)$. We define a similar decomposition for $j_{0}^{K} \widetilde{H}$.

Theorem 3.3. Let $M \subseteq \mathbb{C}^{m+d}$ be of codimension $d$ and of finite type at 0 . Let $M^{\prime} \subseteq \mathbb{C}^{n+e}$ be of codimension e and $k$-nondegenerate at 0 . Then there exists a positive integer $K$ depending only on $M$ and $M^{\prime}$ such that for each $\alpha=\left(\alpha_{1}, \ldots, \alpha_{n}\right)$ with $1 \leq \alpha_{1}<\cdots<\alpha_{n} \leq m$ and each $\beta=\left(\beta_{1}, \ldots, \beta_{n}\right)$ with $1 \leq \beta_{1}<\cdots<\beta_{n} \leq m$, there exists a $\mathbb{C}^{n+e}$-valued holomorphic function defined on an open subset of $\mathbb{C}^{m+d} \times J^{K}\left(\mathbb{C}^{m+d}, \mathbb{C}^{n+e}\right)_{(0,0)} \times J^{K}\left(\mathbb{C}^{m+d}, \mathbb{C}^{n+e}\right)_{(0,0)}$ of the form

$$
\Phi^{\alpha, \beta}(Z, \Lambda, \Gamma)=\sum_{\gamma} \frac{R_{\gamma}^{\alpha, \beta}(\Lambda, \Gamma)}{\left(\operatorname{det}\left(\Lambda^{r, \alpha_{j}}\right)_{1 \leq r, j \leq n}\right)^{s_{\alpha \beta \gamma}}\left(\operatorname{det}\left(\Gamma^{r, \beta_{j}}\right)_{1 \leq r, j \leq n}\right)^{t_{\alpha \beta \gamma}}} Z^{\gamma},
$$

where $R_{\gamma}^{\alpha, \beta}$ are $\mathbb{C}^{n+e}$-valued polynomials and $s_{\alpha \beta \gamma}$ and $t_{\alpha \beta \gamma}$ are nonnegative integers, such that if $\mathscr{H}(Z, \zeta)=(H(Z), \widetilde{H}(\zeta))$ is a germ at 0 of an HSPM satisfying condition $D_{\mu \nu}$, then

$$
\begin{aligned}
H(Z) & =\Phi^{\mu, \nu}\left(Z, j_{0}^{K}(H), j_{0}^{K}(\widetilde{H})\right), \\
\widetilde{H}(\zeta) & =\overline{\Phi^{\nu, \mu}}\left(\zeta, j_{0}^{K}(\widetilde{H}), j_{0}^{K}(H)\right),
\end{aligned}
$$

for $(Z, \zeta)$ sufficiently close to 0 . Furthermore, for any $\left(\Lambda_{0}, \Gamma_{0}\right)$ such that

$$
\operatorname{det}\left(\Lambda_{0}^{r, \alpha_{j}}\right)_{1 \leq r, j \leq n} \neq 0 \quad \text { and } \operatorname{det}\left(\Gamma_{0}^{r, \beta_{j}}\right)_{1 \leq r, j \leq n} \neq 0,
$$

the function $\Phi^{\alpha, \beta}$ is holomorphic in a neighborhood of $\left(0, \Lambda_{0}, \Gamma_{0}\right)$. 
Remark 3.4. It is implicit in the hypotheses of Theorem 3.3 that $m \geq n$. However, if we assume that $m<n$, even if the matrices $\left(f_{z}(0)\right):=\left(f_{z_{l}}^{j}(0)\right)_{1 \leq l \leq m, 1 \leq j \leq n}$ and $\left(\tilde{f}_{\chi}(0)\right):=\left(\tilde{f}_{\chi l}^{j}(0)\right)_{1 \leq l \leq m, 1 \leq j \leq n}$ have maximal rank, the theorem will not hold. Let $M \subseteq \mathbb{C}^{4}$ be defined by $M=\left\{\operatorname{Im} w_{1}=\left|z_{1}\right|^{2}, \operatorname{Im} w_{2}=\left|z_{2}\right|^{2}\right\}$. Let $M^{\prime} \subseteq \mathbb{C}^{4}$ be defined by $M^{\prime}=\left\{\operatorname{Im} w^{\prime}=\left|z_{1}^{\prime}\right|^{2}+\left|z_{2}^{\prime}\right|^{2}+\left|z_{3}^{\prime}\right|^{2}\right\}$. Then $M$ is of finite type at 0 , and $M^{\prime}$ is 1 -nondegenerate at 0 . For any positive integer $r$, define

$$
\begin{aligned}
& \mathscr{H}_{r}(z, w, \chi, \tau) \\
& =\left(z_{1}, z_{2}, w_{1}, w_{1}+w_{2}, \chi_{1}-2 i \chi_{1} \tau_{1}-2 i \chi_{1} \tau_{1}^{r}, \chi_{2}, \tau_{1}^{r}+\tau_{1}, \tau_{1}+\tau_{2}-2 i \tau_{1}^{2}-2 i \tau_{1}^{r+1}\right) .
\end{aligned}
$$

Observe that $\mathcal{H}_{r}$ is an HSPM sending $(\mathcal{M}, 0)$ into $\left(\mathcal{M}^{\prime}, 0\right)$ and is a biholomorphism near 0 .

The proof of Theorem 3.3 will be based on arguments from [Baouendi et al. 1999a; 2001]. Before proving the theorem, we first introduce a few lemmas.

Lemma 3.5. Let $\mathscr{H}(Z, \zeta)=(H(Z), \widetilde{H}(\zeta))$ be an HSPM that sends $(M, 0)$ into $\left(\mathcal{M}^{\prime}, 0\right)$. Then $\mathcal{H}^{\prime}(Z, \zeta)=(\overline{\widetilde{H}}(Z), \bar{H}(\zeta))$ is an HSPM sending $(\mathcal{M}, 0)$ into $\left(\mathcal{M}^{\prime}, 0\right)$.

Proof. Let $\rho=\left(\rho_{1}, \ldots, \rho_{d}\right)$ be a defining function for $M$, and let $\rho^{\prime}=\left(\rho_{1}^{\prime}, \ldots, \rho_{e}^{\prime}\right)$ be a defining function for $M^{\prime}$. For $j=1, \ldots, e$ and $k=1, \ldots, d$, there exist holomorphic functions $a_{k}^{j}$ such that

$$
\begin{aligned}
& \rho_{j}^{\prime}(H(Z), \widetilde{H}(\zeta))=\sum_{k=1}^{d} a_{k}^{j}(Z, \zeta) \rho_{k}(Z, \zeta) \text { implies } \\
& \bar{\rho}_{j}^{\prime}(\widetilde{H}(\zeta), H(Z))=\sum_{k=1}^{d} a_{k}^{j}(Z, \zeta) \rho_{k}(Z, \zeta) \text { implies }
\end{aligned}
$$

$$
\rho_{j}^{\prime}(\overline{\widetilde{H}}(Z), \bar{H}(\zeta))=\sum_{k=1}^{d} \bar{a}_{k}^{j}(\zeta, Z) \bar{\rho}_{k}(\zeta, Z)=\sum_{k=1}^{d} \bar{a}_{k}^{j}(\zeta, Z) \rho_{k}(Z, \zeta) .
$$

Equations (3-7) and (3-8) follow from the reality of the $\rho_{j}$.

The following notation will be used in Lemmas 3.6, 3.7, and 3.10. Let $M, M^{\prime}$, and $\mathcal{H}$ be as in Theorem 3.3. We will write $j_{Z}^{K} H=\left(\left(j_{Z}^{K}\right)^{\prime \prime} H,\left(g_{z^{\alpha}}(Z)\right)_{|\alpha| \leq K}\right)$, where the remaining derivatives of $H$ at $Z$ are represented by $\left(j_{Z}^{K}\right)^{\prime \prime} H$. Given any $\Lambda \in J_{Z}^{K}\left(\mathbb{C}^{m+d}, \mathbb{C}^{n+e}\right)_{(Z, H(Z))}$, we will also write

$$
\Lambda=\left(\Lambda_{1}, \Lambda_{2}\right),
$$

where $\left(j_{Z}^{K} H\right)_{2}$ is exactly $\left(g_{z^{\alpha}}(Z)\right)_{|\alpha| \leq K}$. We do a similar decomposition for $j_{\zeta}^{K} \widetilde{H}$. Lemma 3.6. Let $M$ and $M^{\prime}$ be as in Theorem 3.3. Then for any $\beta=\left(\beta_{1}, \ldots, \beta_{n}\right)$ and $\alpha=\left(\alpha_{1}, \ldots, \alpha_{n}\right)$, with $1 \leq \alpha_{1}<\cdots<\alpha_{n} \leq m$, there exists a $\mathbb{C}^{e}$-valued 
holomorphic function $\phi_{\beta}^{\alpha}$ defined on an open subset of $\mathbb{C}^{K_{\beta}} \times \mathbb{C}^{m+d} \times \mathbb{C}^{m+d}$, for some integer $K_{\beta}$, of the form

$$
\phi_{\beta}^{\alpha}(\Lambda, Z, \zeta)=\sum_{\gamma, \delta, \kappa} \frac{P_{\gamma, \delta, \kappa}^{\alpha, \beta}\left(\Lambda_{1}\right)}{\left(\operatorname{det}\left(\Lambda^{j, \alpha_{l}}\right)_{1 \leq j, l \leq n}\right)^{t_{\alpha \beta \gamma} \delta \kappa}} Z^{\gamma} \zeta^{\delta} \Lambda_{2}^{\kappa},
$$

where $t_{\alpha \beta \gamma \delta \kappa}$ are nonnegative integers, $P_{\gamma, \delta, \kappa}^{\alpha, \beta}$ are $\mathbb{C}^{e}$-valued polynomials, and $\phi_{\beta}^{\alpha}$ identically vanishes whenever $\zeta=0$ and $\Lambda_{2}=0$, such that if $\mathcal{H}$ is a germ at 0 of an HSPM satisfying condition $D_{\mu \nu}$, then for $(Z, \zeta) \in \mathcal{M}$ sufficiently close to 0 ,

$$
\begin{aligned}
Q_{z^{\prime \beta}}^{\prime}(f(Z), \tilde{f}(\zeta), \tilde{g}(\zeta)) & =\phi_{\beta}^{\mu}\left(j_{Z}^{|\beta|}(H), Z, \zeta\right), \\
\bar{Q}_{\chi^{\prime \beta}}^{\prime}(\tilde{f}(\zeta), f(Z), g(Z)) & =\overline{\phi_{\beta}^{v}}\left(j_{\zeta}^{|\beta|}(\widetilde{H}), \zeta, Z\right) .
\end{aligned}
$$

Also $\phi_{\beta}^{\alpha}$ is holomorphic near $\left(\Lambda_{0}, 0,0\right)$ for any $\Lambda_{0}$ such that $\operatorname{det}\left(\Lambda_{0}^{j, \alpha_{l}}\right)_{1 \leq j, l \leq n} \neq 0$. Proof. For $j=1, \ldots, m$,

$$
L_{j}=\frac{\partial}{\partial z_{j}}+\sum_{r=1}^{d} Q_{z_{j}}^{r}(z, \chi, \tau) \frac{\partial}{\partial w_{r}}
$$

are vector fields tangent to $\mathcal{M}$. Let $\hat{z}:=\left(z_{\mu_{1}}, \ldots, z_{\mu_{n}}\right)$. Applying $L_{\mu_{1}}, \ldots, L_{\mu_{n}}$ to

$$
g(z, w)=Q^{\prime}(f(z, w), \widetilde{H}(\chi, \tau))
$$

we get (in matrix notation)

$$
\begin{aligned}
g_{\hat{z}}(z, w)+Q_{\hat{z}}(z, \chi, \tau) g_{w}(z, w) & \\
& =\left(f_{\hat{z}}(z, w)+Q_{\hat{z}}(z, \chi, \tau) f_{w}(z, w)\right) Q_{z^{\prime}}^{\prime}(f(z, w), \widetilde{H}(\chi, \tau))
\end{aligned}
$$

for all $(z, w, \chi, \tau) \in \mathcal{M}$. By assumption, $\left(f_{\hat{z}}(0)\right)$ is invertible, so we have near $(z, w, \chi, \tau)=(0,0,0,0)$,

$$
\begin{aligned}
& Q_{z^{\prime}}^{\prime}(f(z, w), \widetilde{H}(\chi, \tau)) \\
& =\left(f_{\hat{z}}(z, w)+Q_{\hat{z}}(z, \chi, \tau) f_{w}(z, w)\right)^{-1}\left(g_{\hat{z}}(z, w)+Q_{\hat{z}}(z, \chi, \tau) g_{w}(z, w)\right) .
\end{aligned}
$$

We claim that the right hand side of (3-16) can be written in the form

$$
\sum_{\gamma, \delta} \frac{p_{\gamma, \delta}^{\mu}\left(j_{Z}^{1}(H)\right)}{\left(\operatorname{det}\left(f_{\hat{z}}(Z)\right)\right)^{s_{\mu \gamma \delta}}} Z^{\gamma} \zeta^{\delta},
$$

where each $p_{\gamma, \delta}^{\mu}$ is an $n \times e$ polynomial matrix and each $s_{\mu \gamma \delta}$ is a nonnegative integer. This comes from writing the right hand side as

$$
\left(f_{\hat{z}}+Q_{\hat{z}} f_{w}\right)^{-1}\left(g_{\hat{z}}+Q_{\hat{z}} g_{w}\right)=\left(I+f_{\hat{z}}^{-1} f_{w} Q_{\hat{z}}\right)^{-1}\left(f_{\hat{z}}^{-1}\right)\left(g_{\hat{z}}+Q_{\hat{z}} g_{w}\right)
$$


The last factor on the right of (3-18) can clearly be written in the form (3-17), as it is independent of $\operatorname{det}\left(f_{\hat{z}}(Z)\right)$. The second can be written in the form (3-17) since for any invertible matrix $A$, we can write $A^{-1}$ as $(1 / \operatorname{det} A)(\operatorname{adj} A)$. The first can also be written in the form (3-17). Indeed, since $f_{\hat{z}}^{-1}(0) f_{w}(0) Q_{\hat{z}}(0)=0$, then for $(z, \chi, \tau)$ sufficiently close to 0 , we have $(I+B)^{-1}=\sum_{j=0}^{\infty}(-1)^{j} B^{j}$, where we define $B:=f_{\hat{z}}^{-1} f_{w} Q_{\hat{z}}$. We then use the aforementioned formula for the inverse of a matrix, and the claim is proved. Defining $j_{Z}^{1} H=\left(\left(j_{Z}^{1}\right)^{\prime} H,\left(j_{Z}^{1}\right)^{\prime \prime} H\right)$, where $\left(j_{Z}^{1}\right)^{\prime \prime} H=\left(g_{z_{j}}(Z)\right)$ and $\left(j_{Z}^{1}\right)^{\prime} H$ represents the remaining derivatives at $Z$, it immediately follows that (3-17) can be written in the form

$$
\sum_{\gamma, \delta, \kappa} \frac{p_{\gamma, \delta, \kappa}^{\mu}\left(\left(j_{Z}^{1}\right)^{\prime} H\right)}{\left(\operatorname{det}\left(f_{\hat{z}}(Z)\right)\right)^{s_{\mu \gamma \delta \kappa}}} Z^{\gamma} \zeta^{\delta}\left(\left(j_{Z}^{1}\right)^{\prime \prime} H\right)^{\kappa} .
$$

We get (3-11) from (3-16) and (3-19) by inductively applying the $L_{j}$ and using the chain rule. To complete the proof of the lemma, we use Lemma 3.5 to see that $(\bar{H}, \bar{H})$ sends $\mathcal{M}$ into $\mathcal{M}^{\prime}$ and satisfies condition $D_{\nu \mu}$. So as we have seen in this proof,

$$
Q_{z^{\prime \beta}}^{\prime}(\bar{f}(Z), \bar{f}(\zeta), \bar{g}(\zeta))=\phi_{\beta}^{\nu}\left(j_{Z}^{|\beta|}(\overline{\widetilde{H}}), Z, \zeta\right) .
$$

Taking the complex conjugate of this entire equation gives (3-12).

The fact that $\phi_{\beta}^{\alpha} \equiv 0$ whenever $\zeta=0$ and $\Lambda_{2}=0$ follows from (3-16), the definition of the $L_{j}$ given in (3-13), and the fact that $Q_{z^{\beta}}(z, 0,0) \equiv 0$ for any $\beta$.

Lemma 3.7. Let $M$ and $M^{\prime}$ be as in Theorem 3.3. For each $\beta=\left(\beta_{1}, \ldots, \beta_{n+e}\right)$ and $\alpha=\left(\alpha_{1}, \ldots, \alpha_{n}\right)$ with $1 \leq \alpha_{1}<\cdots<\alpha_{n} \leq m$, there exists $a \mathbb{C}^{n+e}$-valued function $\Psi_{\beta}^{\alpha}$, holomorphic on an open subset of $\mathbb{C}^{m+d} \times \mathbb{C}^{m+d} \times \mathbb{C}^{K_{\beta}}$ for some integer $K_{\beta}$, of the form

$$
\Psi_{\beta}^{\alpha}(Z, \zeta, \Lambda)=\sum_{\gamma, \delta, \kappa} \frac{P_{\gamma, \delta, \kappa}^{\alpha, \beta}\left(\Lambda_{1}\right)}{\left(\operatorname{det}\left(\Lambda^{r, \alpha_{l}}\right)_{1 \leq l, r \leq n}\right)^{t_{\alpha \beta \gamma \delta \kappa}}} Z^{\gamma} \zeta^{\delta} \Lambda_{2}^{\kappa},
$$

where $P_{\gamma, \delta, \kappa}^{\alpha, \beta}\left(\Lambda_{1}\right)$ are $\mathbb{C}^{n+e}$-valued polynomials and $t_{\alpha \beta \gamma \delta \kappa}$ are nonnegative integers, such that if $\mathcal{H}$ is a germ at 0 of an HSPM satisfying condition $D_{\mu \nu}$, then for $(Z, \zeta) \in \mathcal{M}$ sufficiently close to 0 ,

$$
\begin{aligned}
\partial^{\beta} H(Z) & =\Psi_{\beta}^{\nu}\left(Z, \zeta, j_{\zeta}^{k+|\beta|}(\widetilde{H})\right), \\
\partial^{\beta} \widetilde{H}(\zeta) & =\overline{\Psi_{\beta}^{\mu}}\left(\zeta, Z, j_{Z}^{k+|\beta|}(H)\right) .
\end{aligned}
$$

Also, $\Psi_{\beta}^{\alpha}$ is holomorphic near $\left(0,0, \Lambda_{0}\right)$ for any $\Lambda_{0}$ such that $\operatorname{det}\left(\Lambda_{0}^{r, \alpha_{l}}\right)_{1 \leq l, r \leq n} \neq 0$.

Proof. As $M^{\prime}$ is $k$-nondegenerate at 0 , assume the vectors $\bar{Q}_{z \chi^{\alpha_{1}}}^{\prime j_{1_{1}}}(0), \ldots, \bar{Q}_{z \chi^{\alpha_{n}}}^{\prime j_{n_{n}}}(0)$ span $\mathbb{C}^{n}$, where each $j_{k} \in\{1, \ldots, e\}$, each $\left|\alpha_{j}\right| \leq k$, and $\bar{Q}^{\prime}=\left(\bar{Q}^{\prime 1}, \ldots, \bar{Q}^{\prime e}\right)$. From 
Lemma 3.6, we have for each $(Z, \zeta) \in \mathcal{M}$

$$
\bar{Q}_{\chi^{\prime \alpha_{1}}}^{j_{1}}(\tilde{f}(\zeta), f(Z), g(Z))=\overline{\left(\phi_{\alpha_{1}}^{\nu}\right)^{j_{1}}}\left(j_{\zeta}^{\left|\alpha_{1}\right|}(\widetilde{H}), \zeta, Z\right)
$$

$$
\bar{Q}_{\chi^{\prime a_{n}}}^{j_{j_{n}}}(\tilde{f}(\zeta), f(Z), g(Z))=\overline{\left(\phi_{\alpha_{n}}^{v}\right)^{j_{n}}}\left(j_{\zeta}^{\left|\alpha_{n}\right|}(\widetilde{H}), \zeta, Z\right),
$$

where $\phi_{\alpha}^{\beta}=\left(\left(\phi_{\alpha}^{\beta}\right)^{1}, \ldots,\left(\phi_{\alpha}^{\beta}\right)^{e}\right)$. Using this system of equations, coupled with the fact that normal coordinates for $M^{\prime}$ imply that $\bar{Q}^{\prime}\left(\chi^{\prime}, 0, w^{\prime}\right) \equiv \bar{Q}^{\prime}\left(0, z^{\prime}, w^{\prime}\right) \equiv w^{\prime}$, we can apply the implicit function theorem to find a map $B^{v}: \mathbb{C}^{n} \times \mathbb{C}^{n} \rightarrow \mathbb{C}^{n+e}$, holomorphic near 0 , such that

$$
H(Z)=B^{v}\left(\tilde{f}(\zeta),\left(\overline{\left(\phi_{\alpha_{l}}^{v}\right)^{j_{l}}}\left(j_{\zeta}^{\left|\alpha_{l}\right|} \widetilde{H}, \zeta, Z\right)\right)_{1 \leq l \leq n}\right) .
$$

If we Taylor expand, we can write the right hand side of (3-25) as

$$
\sum_{\alpha, \beta, \gamma} A_{\alpha \beta \gamma}^{v}\left(\widetilde{\Lambda}_{1}\right) Z^{\alpha} \zeta^{\beta} \widetilde{\Lambda}_{2}^{\gamma}
$$

where we remind the reader that $\widetilde{\Lambda}_{2}$ corresponds to $\left(\tilde{g}_{\chi^{\alpha}}(\zeta)\right)$, and $\widetilde{\Lambda}_{1}$ corresponds to the remaining derivatives of $\widetilde{H}$ at $\zeta$. We claim that each $A_{\alpha \beta \gamma}^{v}$ is rational. This follows from the fact that $\tilde{f}(0)=0$, the form of $\phi_{\beta}^{\alpha}$ as given in (3-10), and the fact that $\phi_{\beta}^{\alpha} \equiv 0$ whenever $\zeta=0$ and $\Lambda_{2}=0$ (refer to the statement of Lemma 3.6).

Furthermore, each $A_{\alpha \beta \gamma}^{v}\left(\widetilde{\Lambda}_{1}\right)$ is of the form given in the right hand side of (3-21). This can be seen by Taylor expanding $B^{v}$ as given in (3-25) and plugging in (3-10). Define

$$
\Psi_{0}^{v}(Z, \zeta, \widetilde{\Lambda}):=\sum_{\alpha, \beta, \gamma} A_{\alpha \beta \gamma}^{v}\left(\widetilde{\Lambda}_{1}\right) Z^{\alpha} \zeta^{\beta} \widetilde{\Lambda}_{2}^{\gamma}
$$

This proves (3-22) for $|\beta|=0$. For $|\beta|>0$, as every point in $\mathcal{M}$ is of the form $(z, w, \chi, \bar{Q}(\chi, z, w))$, we have

$$
H(z, w) \equiv \Psi_{0}^{v}\left(z, w, \chi, \bar{Q}(\chi, z, w), j_{(\chi, \bar{Q}(\chi, z, w))}^{k}(\widetilde{H})\right) .
$$

We inductively differentiate (3-28), applying the chain rule, and (3-22) follows.

To get (3-23), we know from Lemma 3.5 that $(\bar{H}, \bar{H})$ sends $\mathcal{M}$ into $\mathcal{M}^{\prime}$ and satisfies condition $D_{\nu \mu}$. So

$$
\partial^{\beta} \widetilde{H}(Z)=\Psi_{\beta}^{\mu}\left(Z, \zeta, j_{\zeta}^{k+|\beta|}(\bar{H})\right) .
$$

Taking the complex conjugate of both sides of this equation, the lemma follows.

Now we define the $r$-th Segre mappings of $M$ at 0 . These mappings were first introduced by Baouendi, Ebenfelt, and Rothschild [1996] and will prove extremely 
useful in completing the proof of Theorem 3.3. Given a positive integer $r$, let $t^{0}, \ldots, t^{r-1} \in \mathbb{C}^{m}$ and define $v^{r}: \mathbb{C}^{r m} \rightarrow \mathbb{C}^{m+d}$ by

$$
v^{r}\left(t^{0}, \ldots, t^{r-1}\right):=\left(t^{0}, u^{r}\left(t^{0}, \ldots, t^{r-1}\right)\right),
$$

where $u^{r}: \mathbb{C}^{r m} \rightarrow \mathbb{C}^{d}$ is given inductively by

$$
u^{1}\left(t^{0}\right)=0, \quad u^{r}\left(t^{0}, \ldots, t^{r-1}\right)=Q\left(t^{0}, t^{1}, \overline{u^{r-1}}\left(t^{1}, \ldots, t^{r-1}\right)\right) \text { for } r \geq 2 .
$$

Definition 3.8. Let $V$ and $W$ be finite-dimensional complex vector spaces. Let $\mathscr{R}_{0}(V \times W, V)$ denote the ring of germs of holomorphic functions $f$ at $V \times\{0\}$ in $V \times W$ which can be written in the form $f(\Lambda, \Gamma)=\sum_{\alpha} p_{\alpha}(\Lambda) \Gamma^{\alpha}$, where each $p_{\alpha}(\Lambda)$ is a polynomial function on $V$.

The following lemma is proved in [Baouendi et al. 2001]:

Lemma 3.9. Let $V_{0}, V_{1}, \widetilde{V}_{0}, \widetilde{V}_{1}$ be finite-dimensional complex vector spaces with fixed bases and $x_{0}, x_{1}, \tilde{x}_{0}, \tilde{x}_{1}$ be the linear coordinates with respect to these bases. Let $p \in \mathbb{C}\left[x_{0}\right]$ and $\tilde{p} \in \mathbb{C}\left[\tilde{x}_{0}\right]$ be nontrivial polynomial functions on $V_{0}$ and $\widetilde{V}_{0}$ respectively, and let

$$
\phi=\left(\phi_{0}, \phi_{1}\right): \mathbb{C} \times V_{0} \times V_{1} \rightarrow \widetilde{V}_{0} \times \widetilde{V}_{1}
$$

be a germ of a holomorphic map with components in $\mathscr{R}_{0}\left(\mathbb{C} \times V_{0} \times V_{1}, \mathbb{C} \times V_{0}\right)$, such that $\phi\left(\mathbb{C} \times V_{0} \times\{0\}\right) \subseteq \widetilde{V}_{0} \times\{0\}$, and satisfying $\tilde{p}\left(\phi_{0}\left(1 / p\left(x_{0}\right), x_{0}, 0\right)\right) \not \equiv 0$. Then given any $\tilde{h} \in \mathscr{R}_{0}\left(\mathbb{C} \times \widetilde{V}_{0} \times \widetilde{V}_{1}, \mathbb{C} \times \widetilde{V}_{0}\right)$, there exists $h \in \mathscr{R}_{0}\left(\mathbb{C} \times V_{0} \times V_{1}, \mathbb{C} \times V_{0}\right)$ such that

$$
\tilde{h}\left(\frac{1}{\tilde{p}\left(\phi_{0}\left(1 / p\left(x_{0}\right), x_{0}, x_{1}\right)\right)}, \phi\left(\frac{1}{p\left(x_{0}\right)}, x_{0}, x_{1}\right)\right) \equiv h\left(\frac{1}{q\left(x_{0}\right)}, x_{0}, x_{1}\right),
$$

with $q\left(x_{0}\right):=p\left(x_{0}\right)^{t} \tilde{p}\left(\phi_{0}\left(1 / p\left(x_{0}\right), x_{0}, 0\right)\right)$ for some positive integer $t$. Also, $h$ vanishes on $\mathbb{C} \times V_{0} \times\{0\}$ if $\tilde{h}$ vanishes on $\mathbb{C} \times \widetilde{V}_{0} \times\{0\}$.

Lemma 3.9 will be key in establishing the following lemma.

Lemma 3.10. Let $M$ and $M^{\prime}$ be as in Theorem 3.3. Given any $\beta=\left(\beta_{1}, \ldots, \beta_{n}\right)$ and $\alpha=\left(\alpha_{1}, \ldots, \alpha_{n}\right)$, with $1 \leq \beta_{1}<\cdots<\beta_{n} \leq m$ and $1 \leq \alpha_{1}<\cdots<\alpha_{n} \leq m$, and any positive integer $s$, there exists $a \mathbb{C}^{n+e}$-valued function $\Xi_{s}^{\alpha, \beta}(x, \Lambda, \Gamma)$ holomorphic on an open subset of $\mathbb{C}^{s m} \times J^{s k}\left(\mathbb{C}^{m+d}, \mathbb{C}^{n+e}\right)_{(0,0)} \times J^{s k}\left(\mathbb{C}^{m+d}, \mathbb{C}^{n+e}\right)_{(0,0)}$ of the form

$$
\Xi_{s}^{\alpha, \beta}(x, \Lambda, \Gamma)=\sum_{\gamma} \frac{P_{\gamma}^{\alpha \beta s}(\Lambda, \Gamma)}{Q_{\gamma}^{\alpha \beta s}(\Lambda, \Gamma)} x^{\gamma},
$$

where each $P_{\gamma}^{\alpha \beta s}$ is a $\mathbb{C}^{n+e}$-valued polynomial, and

$$
Q_{\gamma}^{\alpha \beta s}(\Lambda, \Gamma):=\left(\operatorname{det}\left(\Lambda^{r, \alpha_{l}}\right)_{1 \leq r, l \leq n}\right)^{u_{\alpha \beta \gamma s}}\left(\operatorname{det}\left(\Gamma^{r, \beta_{l}}\right)_{1 \leq r, l \leq n}\right)^{v_{\alpha \beta \gamma s}}
$$


for some nonnegative integers $u_{\alpha \beta \gamma s}$ and $v_{\alpha \beta \gamma s}$, such that if $\mathcal{H}$ is a germ at 0 of an $H S P M$ satisfying condition $D_{\mu \nu}$, then

$$
\begin{aligned}
& H\left(v^{s}\left(t^{0}, \ldots, t^{s-1}\right)\right)=\Xi_{s}^{\mu, \nu}\left(t^{0}, \ldots, t^{s-1}, j_{0}^{s k} H, j_{0}^{s k} \widetilde{H}\right), \\
& \widetilde{H}\left(\overline{v^{s}}\left(t^{0}, \ldots, t^{s-1}\right)\right)=\bar{\Xi}_{s}^{v, \mu}\left(t^{0}, \ldots, t^{s-1}, j_{0}^{s k} \widetilde{H}, j_{0}^{s k} H\right),
\end{aligned}
$$

for $t^{0}, \ldots, t^{s-1}$ sufficiently close to 0 . Furthermore, for any $\left(\Lambda_{0}, \Gamma_{0}\right)$ such that $\operatorname{det}\left(\Lambda_{0}^{r, \alpha_{l}}\right)_{1 \leq r, l \leq n} \neq 0$ and $\operatorname{det}\left(\Gamma_{0}^{r, \beta_{l}}\right)_{1 \leq r, l \leq n} \neq 0$, the function $\Xi_{s}^{\alpha, \beta}$ is holomorphic on a neighborhood of $\left(0, \Lambda_{0}, \Gamma_{0}\right)$.

Proof. We inductively prove something stronger. First, we simplify notation slightly. Define

$$
\begin{aligned}
& p^{\alpha}\left(\Lambda_{1}(Z)\right):=\operatorname{det}\left(\Lambda^{r, \alpha_{l}}(Z)\right)_{1 \leq r, l \leq n}, \\
& \tilde{p}^{\beta}\left(\widetilde{\Lambda}_{1}(\zeta)\right):=\operatorname{det}\left(\widetilde{\Lambda}^{r, \beta_{l}}(\zeta)\right)_{1 \leq r, l \leq n},
\end{aligned}
$$

where $\Lambda_{1}$ is as defined in (3-9) (and $\widetilde{\Lambda}_{1}$ is defined similarly). We will show that for any $\gamma$ and $s$, there exist nonnegative integers $a_{\alpha \beta}^{s}, b_{\alpha \beta}^{s}$ and holomorphic maps $\Theta_{s}^{\alpha, \beta, \gamma}$ with components in

$$
\begin{aligned}
\mathscr{R}_{0}\left(\mathbb{C} \times J^{k s+|\gamma|}\left(\mathbb{C}^{m+d},\right.\right. & \left.\mathbb{C}^{n+e}\right)_{(0,0)} \times J^{k s+|\gamma|}\left(\mathbb{C}^{m+d}, \mathbb{C}^{n+e}\right)_{(0,0)} \times \mathbb{C}^{m s}, \\
& \left.\mathbb{C} \times J^{k s+|\gamma|}\left(\mathbb{C}^{m+d}, \mathbb{C}^{n+e}\right)_{(0,0)} \times J^{k s+|\gamma|}\left(\mathbb{C}^{m+d}, \mathbb{C}^{n+e}\right)_{(0,0)}\right)
\end{aligned}
$$

such that

$$
\begin{aligned}
& \partial^{\gamma} H\left(v^{s}\left(t^{0}, \ldots, t^{s-1}\right)\right) \\
= & \Theta_{s}^{\mu, \nu, \gamma}\left(\frac{1}{p^{\mu}\left(\Lambda_{1}(0)\right)^{a_{\mu \nu}^{s}} \tilde{p}^{\nu}\left(\widetilde{\Lambda}_{1}(0)\right)^{b_{\mu \nu}^{s}}}, j_{0}^{k s+|\gamma|} H, j_{0}^{k s+|\gamma|} \widetilde{H}, t^{0}, \ldots, t^{s-1}\right)
\end{aligned}
$$

and

$$
\begin{aligned}
& \partial^{\gamma} \widetilde{H}\left(\overline{v^{s}}\left(t^{0}, \ldots, t^{s-1}\right)\right) \\
= & \overline{\Theta_{s}^{v, \mu, \gamma}}\left(\frac{1}{p^{\nu}\left(\widetilde{\Lambda}_{1}(0)\right)^{a_{\nu \mu}^{s}} \tilde{p}^{\mu}\left(\Lambda_{1}(0)\right)^{b_{\nu \mu}^{s}}}, j_{0}^{k s+|\gamma|} \widetilde{H}, j_{0}^{k s+|\gamma|} H, t^{0}, \ldots, t^{s-1}\right) .
\end{aligned}
$$

First, we reformulate Lemma 3.7 with new notation. Let $j_{Z}^{l} H=\left(\left(j_{Z}^{l}\right)^{\prime} H,\left(j_{Z}^{l}\right)^{\prime \prime} H\right)$, where $\left(j_{Z}^{l}\right)^{\prime \prime} H=\left(g_{z^{\alpha}}(Z)\right)_{|\alpha| \leq l}$, and $\left(j_{Z}^{l}\right)^{\prime} H$ represents the remaining derivatives at $Z$. (A similar decomposition applies to $\widetilde{H}$ ). According to Lemma 3.7, there exist maps $\theta_{\gamma}^{\alpha}$ with components in $\Re_{0}\left(\mathbb{C} \times \mathbb{C}^{l_{\gamma}^{\prime}} \times \mathbb{C}^{2 m+2 d} \times \mathbb{C}^{l_{\gamma}^{\prime \prime}}, \mathbb{C} \times \mathbb{C}_{\gamma}^{l_{\gamma}^{\prime}}\right)$, for some integers $l_{\gamma}^{\prime}$ and $l_{\gamma}^{\prime \prime}$, such that for $(Z, \zeta) \in \mathcal{M}$,

$$
\begin{aligned}
\partial^{\gamma} H(Z) & =\theta_{\gamma}^{\nu}\left(\frac{1}{\tilde{p}^{\nu}\left(\left(j_{\zeta}^{k+|\gamma|}\right)^{\prime} \widetilde{H}\right)},\left(j_{\zeta}^{k+|\gamma|}\right)^{\prime} \widetilde{H}, Z, \zeta,\left(j_{\zeta}^{k+|\gamma|}\right)^{\prime \prime} \widetilde{H}\right), \\
\partial^{\gamma} \widetilde{H}(\zeta) & =\overline{\theta_{\gamma}^{\mu}}\left(\frac{1}{p^{\mu}\left(\left(j_{Z}^{k+|\gamma|}\right)^{\prime} H\right)},\left(j_{Z}^{k+|\gamma|}\right)^{\prime} H, \zeta, Z,\left(j_{Z}^{k+|\gamma|}\right)^{\prime \prime} H\right) .
\end{aligned}
$$


It is easy to show that (3-36) and (3-37) hold for $s=1$ by letting $(Z, \zeta)=((z, 0), 0)$ in (3-38) and $(Z, \zeta)=(0,(\chi, 0))$ in (3-39). So now assume (3-36) and (3-37) hold for $s$ replaced by $s-1$, for some $s>1$. We will show they hold for $s$.

For any $s$, it is clear from the definition of the Segre mappings that

$$
\left(v^{s}\left(t^{0}, \ldots, t^{s-1}\right), \overline{v^{s-1}}\left(t^{1}, \ldots, t^{s-1}\right)\right) \in M .
$$

Using this fact in (3-38), we see that

$$
\begin{aligned}
& \partial^{\gamma} H\left(v^{s}\left(t^{0}, \ldots, t^{s-1}\right)\right) \\
& =\theta_{\gamma}^{v}\left(\frac{1}{\left.\tilde{p}^{v}\left(j \frac{k+|\gamma|}{v^{s-1}\left(t^{1}, \ldots, t^{s-1}\right)}\right)^{\prime} \widetilde{H}\right)},\left(j \frac{k+|\gamma|}{v^{s-1}\left(t^{1}, \ldots, t^{s-1}\right)}\right)^{\prime} \widetilde{H},\right. \\
& \left.\qquad v^{s}\left(t^{0}, \ldots, t^{s-1}\right), \overline{v^{s-1}}\left(t^{1}, \ldots, t^{s-1}\right),\left(j \frac{k+|\gamma|}{v^{s-1}\left(t^{1}, \ldots, t^{s-1}\right)}\right)^{\prime \prime} \widetilde{H}\right) .
\end{aligned}
$$

But by our induction hypothesis,

$$
\begin{aligned}
& j \frac{k+|\gamma|}{v^{s-1}\left(t^{1}, \ldots, t^{s-1}\right)} \widetilde{H} \\
& =\left(\overline { \Theta _ { s - 1 } ^ { \nu , \mu , \Delta } } \left(\frac{1}{\tilde{p}^{\nu}\left(\widetilde{\Lambda}_{1}(0)\right)^{a_{\nu \mu}^{s-1}} p^{\mu}\left(\Lambda_{1}(0)\right)^{b_{\nu \mu}^{s-1}}}, j_{0}^{k s+|\Delta|-k} H,\right.\right. \\
& \left.\left.j_{0}^{k s+|\Delta|-k} \widetilde{H}, t^{1}, \ldots, t^{s-1}\right)\right)_{|\Delta| \leq k+|\gamma|} .
\end{aligned}
$$

For convenience, we write the tuple on the right hand side of the above as $(A, B)$ where $B$ corresponds to $\left.\left(\tilde{g}_{\chi^{\alpha}} \overline{v^{s-1}}\left(t^{1}, \ldots, t^{s-1}\right)\right)\right)_{|\alpha| \leq k+|\gamma|}$, and $A$ corresponds to the remainder. We plug the last equation into the previous to get

$$
\partial^{\gamma} H\left(v^{s}\left(t^{0}, \ldots, t^{s-1}\right)\right)=\theta_{\gamma}^{v}\left(\frac{1}{\tilde{p}^{\nu}(A)}, A, v^{s}\left(t^{0}, \ldots, t^{s-1}\right), \overline{v^{s-1}}\left(t^{1}, \ldots, t^{s-1}\right), B\right) \text {. }
$$

Thus, (3-36) follows from Lemma 3.9.

To finish the proof, we need only show (3-37). Here we apply Lemma 3.5, which tells us that $(\widetilde{H}, \bar{H})$ sends $\mathcal{M}$ into $\mathcal{M}^{\prime}$ and satisfies condition $D_{\nu \mu}$. So by (3-36), we see that

$$
\begin{aligned}
& \partial^{\gamma} \widetilde{\widetilde{H}}\left(v^{s}\left(t^{0}, \ldots, t^{s-1}\right)\right) \\
& \quad=\Theta_{s}^{\nu, \mu, \gamma}\left(\frac{1}{p^{\nu}\left(\bar{\Lambda}_{1}(0)\right)^{a_{\nu \mu}^{s}} \tilde{p}^{\mu}\left(\bar{\Lambda}_{1}(0)\right)^{b_{\nu \mu}^{s}}}, j_{0}^{k s+|\gamma|} \overline{\widetilde{H}}, j_{0}^{k s+|\gamma|} \bar{H}, t^{0}, \ldots, t^{s-1}\right) .
\end{aligned}
$$

As $p^{v}$ and $\tilde{p}^{\mu}$ are polynomials with real coefficients, we take the complex conjugate of both sides of the above to see that (3-37) holds true.

We are almost ready to complete the proof of Theorem 3.3. First, however, we present three lemmas. Lemma 3.11 can be found (using slightly different language) in [Baouendi et al. 1999a] and is thus presented here without proof. Lemma 3.12 
is a generalization of a lemma found in [Baouendi et al. 2001]. Lemma 3.13 can be found in [Baouendi et al. 2001] and is presented here without proof.

Lemma 3.11. Let $M$ be as in Theorem 3.3. Then there exists an integer $r$ such that the matrix

$$
\left(\frac{\partial v^{2 r}}{\partial\left(t^{0}, t^{r+1}, t^{r+2}, \ldots, t^{2 r-1}\right)}\left(0, x^{1}, \ldots, x^{r-1}, x^{r}, x^{r-1}, \ldots, x^{1}\right)\right)
$$

has rank $m+d$ for all $\left(x^{1}, \ldots, x^{r}\right) \in U \backslash V$, where $U \subseteq \mathbb{C}^{r m}$ is an open neighborhood of the origin, and $V$ is a proper holomorphic subvariety of $U$. In addition,

$$
v^{2 r}\left(0, x^{1}, \ldots, x^{r-1}, x^{r}, x^{r-1}, \ldots, x^{1}\right) \equiv 0 .
$$

(Here, $v^{2 r}$ is as defined in (3-30).)

Lemma 3.12. Let $V:\left(\mathbb{C}^{r_{1}} \times \mathbb{C}^{r_{2}}, 0\right) \rightarrow\left(\mathbb{C}^{N}, 0\right), r_{2} \geq N$, be a holomorphic map defined near 0 satisfying $\left.V(x, \xi)\right|_{\xi=0} \equiv 0$, with $(x, \xi) \in \mathbb{C}^{r_{1}} \times \mathbb{C}^{r_{2}}$, and assume the matrix $((\partial V / \partial \xi)(x, 0))$ has an $N \times N$ minor that is not identically 0 . Then there exist holomorphic maps (defined near 0 )

$$
\delta:\left(\mathbb{C}^{r_{1}}, 0\right) \rightarrow \mathbb{C} \text { and } \phi:\left(\mathbb{C}^{r_{1}} \times \mathbb{C}^{N}, 0\right) \rightarrow\left(\mathbb{C}^{r_{2}}, 0\right)
$$

with $\delta(x) \not \equiv 0$, such that

$$
V\left(x, \phi\left(x, \frac{Z}{\delta(x)}\right)\right) \equiv Z
$$

for all $(x, Z) \in \mathbb{C}^{r_{1}} \times \mathbb{C}^{N}$ such that $\delta(x) \neq 0$ and both $x$ and $Z / \delta(x)$ are sufficiently small. Furthermore, if $V$ is holomorphic algebraic, then given any sufficiently small $x_{0}$ satisfying $\delta\left(x_{0}\right) \neq 0$, the map $\varphi_{x_{0}}(Z):=\phi\left(x_{0}, Z / \delta\left(x_{0}\right)\right)$ is holomorphic algebraic for all $Z$ in a neighborhood of 0 .

Proof. Write $\xi=\left(\xi^{\prime}, \xi^{\prime \prime}\right)$, where

$$
\xi^{\prime}=\left(\xi_{1}, \ldots, \xi_{N}\right) \in \mathbb{C}^{N} \text { and } \xi^{\prime \prime}=\left(\xi_{N+1}, \ldots, \xi_{r_{2}}\right) \in \mathbb{C}^{r_{2}-N} .
$$

Assume, without loss of generality, that $\operatorname{det}\left(\left(\partial V / \partial \xi^{\prime}\right)(x, 0)\right) \not \equiv 0$. We wish to solve the equation $Z=V\left(x, \xi^{\prime}, 0\right)$ for $\xi^{\prime}$. Since $V(x, 0) \equiv 0$, we can write $Z=V\left(x, \xi^{\prime}, 0\right)=a\left(x, \xi^{\prime}\right) \xi^{\prime}$, where $a\left(x, \xi^{\prime}\right)$ is an $N \times N$ matrix of holomorphic functions defined near 0 . By expanding $a\left(x, \xi^{\prime}\right)$, we can write

$$
Z=V\left(x, \xi^{\prime}, 0\right)=a(x, 0) \xi^{\prime}+\left(\left(\xi^{\prime}\right)^{T} R_{j}\left(x, \xi^{\prime}\right) \xi^{\prime}\right)_{1 \leq j \leq N},
$$

where each $R_{j}\left(x, \xi^{\prime}\right)$ is an $N \times N$ matrix of holomorphic functions defined near 0 . Define $d(x):=\operatorname{det}\left(\left(\partial V / \partial \xi^{\prime}\right)(x, 0)\right)$. Using the fact that $(\operatorname{adj}(A)) A=\operatorname{det}(A) I$ 
for any square matrix $A$, we multiply the far left and far right sides of (3-45) by $b(x):=\operatorname{adj}(a(x, 0))$, noting that $a(x, 0)=\left(\partial V / \partial \xi^{\prime}\right)(x, 0)$, to get

$$
b(x) Z-d(x) \xi^{\prime}-b(x)\left(\left(\xi^{\prime}\right)^{T} R_{j}\left(x, \xi^{\prime}\right) \xi^{\prime}\right)_{1 \leq j \leq N}=0 .
$$

Divide both sides of (3-46) by $d(x)^{2}$ and substitute $\tilde{\xi}^{\prime}=\xi^{\prime} / d(x)$ and $\widetilde{Z}=Z / d(x)^{2}$ to get

$$
b(x) \widetilde{Z}-\tilde{\xi}^{\prime}-b(x)\left(\left(\tilde{\xi}^{\prime}\right)^{T} R_{j}\left(x, d(x) \tilde{\xi}^{\prime}\right) \tilde{\xi}^{\prime}\right)_{1 \leq j \leq N}=0 .
$$

By the implicit function theorem, there is a unique holomorphic solution $\tilde{\xi}^{\prime}=$ $\theta(x, \widetilde{Z})$ defined near 0 such that $\theta(0)=0$. Thus, the first part of the theorem follows by letting $\delta(x):=d(x)^{2}$ and

$$
\phi(x, y):=(d(x) \theta(x, y), 0) \quad \text { for }(x, y) \in \mathbb{C}^{r_{1}} \times \mathbb{C}^{N} .
$$

If $V$ is algebraic, the last part of the theorem follows from the algebraic implicit function theorem; see [Baouendi et al. 1999b] for example.

Lemma 3.13. Let $V_{0}$ and $V_{1}$ be finite-dimensional vector spaces with fixed linear coordinates $x_{0}$ and $x_{1}$, respectively. Let $P\left(x_{0}, x_{1}, \lambda\right) \in \mathscr{R}_{0}\left(V_{0} \times V_{1} \times \mathbb{C}, V_{0}\right)$ with $P\left(x_{0}, 0,0\right) \equiv 0$. For a given integer $l \geq 0$, consider the Laurent series expansion

$$
P\left(x_{0}, \frac{x_{1}}{\lambda^{l}}, \lambda\right)=\sum_{\nu \in \mathbb{Z}} c_{v}\left(x_{0}, x_{1}\right) \lambda^{v} .
$$

Then $c_{0}\left(x_{0}, 0\right) \equiv 0$, and $c_{v} \in \mathscr{R}_{0}\left(V_{0} \times V_{1}, V_{0}\right)$ for every $v \in \mathbb{Z}$.

Proof of Theorem 3.3. Let $r$ be as in Lemma 3.11. Take $x=\left(x^{1}, \ldots, x^{r}\right) \in \mathbb{C}^{r m}$ and $y=\left(y^{0}, \ldots, y^{r-1}\right) \in \mathbb{C}^{r m}$. Let $L(x, y):=\left(y^{0}, x^{1}, \ldots, x^{r}, x^{r-1}+y^{r-1}, \ldots, x^{1}+y^{1}\right)$ and $V(x, y):=v^{2 r}(L(x, y))$. In Lemma 3.12, we take $r_{1}=r_{2}=r m$. From (3-42), we see that $V(x, 0) \equiv 0$. Also, from Lemma 3.11, we see that the other hypothesis of Lemma 3.12 holds. Thus we apply Lemma 3.12. Let $\delta$ and $\phi$ be as given in the lemma. We plug these into (3-34) to see that

$$
H(Z) \equiv \Xi_{2 r}^{\mu, v}\left(L\left(x, \phi\left(x, \frac{Z}{\delta(x)}\right)\right), j_{0}^{2 r k} H, j_{0}^{2 r k} \widetilde{H}\right) .
$$

We rewrite the right hand side of (3-48) as

$$
H(Z) \equiv \widehat{\Xi}_{2 r}^{\mu, v}\left(j_{0}^{2 r k} H, j_{0}^{2 r k} \widetilde{H}, \frac{Z}{\delta(x)}, x\right),
$$

noting that the components of

$$
\widehat{\Xi}_{2 r}^{\mu, \nu}: J^{2 r k}\left(\mathbb{C}^{m+d}, \mathbb{C}^{n+e}\right)_{(0,0)} \times J^{2 r k}\left(\mathbb{C}^{m+d}, \mathbb{C}^{n+e}\right)_{(0,0)} \times \mathbb{C}^{m+d} \times \mathbb{C}^{r m} \rightarrow \mathbb{C}^{n+e}
$$

are holomorphic on an open neighborhood of

$$
J^{2 r k}\left(\mathbb{C}^{m+d}, \mathbb{C}^{n+e}\right)_{(0,0)} \times J^{2 r k}\left(\mathbb{C}^{m+d}, \mathbb{C}^{n+e}\right)_{(0,0)} \times \mathbb{C}^{m+d} \times \mathbb{C}^{r m} .
$$


Now choose $x_{0} \in \mathbb{C}^{r m}$ such that $\hat{\delta}(t):=\delta\left(t x_{0}\right) \not \equiv 0$, for $t \in \mathbb{C}$. Since $H(Z)$ is independent of $x$, we can replace $x=t x_{0}$ in (3-49). There exists a smallest integer $l$ such that $\left(d^{l} / d t^{l}\right) \hat{\delta}(0) \neq 0$. To make our calculations easier, consider a holomorphic change of variable $\lambda=h(t)$ near the origin in $\mathbb{C}$, where $h$ is determined by $\delta\left(t x_{0}\right)=\lambda^{l}$. So we now have

$$
\begin{aligned}
\widehat{\widehat{\Xi}}_{2 r}^{\mu, \nu}\left(j_{0}^{2 r k} H, j_{0}^{2 r k} \widetilde{H}, \frac{Z}{\lambda^{l}}, \lambda\right) & :=\widehat{\Xi}_{2 r}^{\mu, \nu}\left(j_{0}^{2 r k} H, j_{0}^{2 r k} \widetilde{H}, \frac{Z}{\lambda^{l}}, x_{0} h^{-1}(\lambda)\right) \\
& \equiv H(Z) .
\end{aligned}
$$

Observe that the components of $\widehat{\Xi}_{2 r}^{\mu, v}$ are in

$$
\begin{aligned}
\mathscr{R}_{0}\left(J^{2 r k}\left(\mathbb{C}^{m+d}, \mathbb{C}^{n+e}\right)_{(0,0)} \times J^{2 r k}\left(\mathbb{C}^{m+d}, \mathbb{C}^{n+e}\right)_{(0,0)} \times \mathbb{C}^{m+d} \times \mathbb{C},\right. \\
\left.J^{2 r k}\left(\mathbb{C}^{m+d}, \mathbb{C}^{n+e}\right)_{(0,0)} \times J^{2 r k}\left(\mathbb{C}^{m+d}, \mathbb{C}^{n+e}\right)_{(0,0)}\right) .
\end{aligned}
$$

To conclude the proof, we expand the left hand side of (3-50) as a Laurent series in $\lambda$. Since $H(Z)$ is independent of $\lambda$, we can let $H(Z)$ be the constant term of the Laurent series. By Lemma 3.13 and the form of $\Xi_{2 r}^{\mu, v}$ given in (3-33), we see that this is exactly of the form (3-3).

Applying Lemma 3.5 , we see that $(\widetilde{H}, \bar{H})$ sends $\mathcal{M}$ into $\mathcal{M}^{\prime}$ and satisfies condition $D_{v \mu}$. From (3-4), we have

$$
\widetilde{H}(Z)=\Phi^{v, \mu}\left(Z, j_{0}^{K}(\widetilde{H}), j_{0}^{K}(\bar{H})\right) .
$$

Take the complex conjugate of this entire equation, and (3-5) follows.

\section{Reformulation of Theorem 1.6.}

Theorem 3.14. Let $M$ and $M^{\prime}$ be as in Theorem 3.3. Then there exists a positive integer $L$, depending only on $M$ and $M^{\prime}$, such that for each $\alpha=\left(\alpha_{1}, \ldots, \alpha_{n}\right)$ with $1 \leq \alpha_{1}<\cdots<\alpha_{n} \leq m$ and each $\beta=\left(\beta_{1}, \ldots, \beta_{n}\right)$ with $1 \leq \beta_{1}<\cdots<\beta_{n} \leq m$, there exist $\mathbb{C}^{2 n+2 e}$-valued holomorphic functions $\Phi_{1}^{\alpha, \beta}$ and $\Phi_{2}^{\alpha, \beta}$ defined on an open subset of $\mathbb{C}^{m+d} \times \mathbb{C}^{m+d} \times J^{L}\left(\mathbb{C}^{m+d}, \mathbb{C}^{n+e}\right)_{(0,0)}$ such that if $\mathscr{H}$ is a germ at 0 of an HSPM satisfying condition $D_{\mu \nu}$, then

$$
\begin{aligned}
& \mathscr{H}(Z, \zeta)=(H(Z), \widetilde{H}(\zeta))=\Phi_{1}^{\mu, v}\left(Z, \zeta, j_{0}^{L} H\right), \\
& \mathscr{H}(Z, \zeta)=(H(Z), \widetilde{H}(\zeta))=\Phi_{2}^{\mu, v}\left(Z, \zeta, j_{0}^{L} \widetilde{H}\right),
\end{aligned}
$$

for $(Z, \zeta)$ sufficiently close to 0.

Proof. We will prove (3-52); the proof of (3-53) follows similarly. We will show inductively that there exist $\mathbb{C}^{n+e}$-valued holomorphic functions $B_{s}^{\alpha, \beta, \gamma}$ defined on 
an open subset of $J^{k s+|\gamma|}\left(\mathbb{C}^{m+d}, \mathbb{C}^{n+e}\right)_{(0,0)} \times \mathbb{C}^{m s}$, such that

$$
\begin{aligned}
& \partial^{\gamma} H\left(v^{s}\left(t^{0}, \ldots, t^{s-1}\right)\right)=B_{s}^{\mu, \nu, \gamma}\left(j_{0}^{k s+|\gamma|} \varphi_{,} t^{0}, \ldots, t^{s-1}\right), \\
& \partial^{\gamma} \widetilde{H}\left(\overline{v^{s}}\left(t^{0}, \ldots, t^{s-1}\right)\right)=\overline{B_{s}^{\nu, \mu, \gamma}}\left(j_{0}^{k s+|\gamma|} \mathscr{G}^{\prime}, t^{0}, \ldots, t^{s-1}\right),
\end{aligned}
$$

where $\varphi=H$ and $\varphi^{\prime}=\widetilde{H}$ if $s$ is even, and $\varphi=\widetilde{H}$ and $\varphi^{\prime}=H$ if $s$ is odd.

For $s=1$, we see that (3-54) and (3-55) hold true by letting $(Z, \zeta)=((z, 0), 0)$ in (3-22) and $(Z, \zeta)=(0,(\chi, 0))$ in (3-23). For some $s>1$, assume (3-54) and (3-55) hold for $s-1$. Assume, without loss of generality, that $s$ is even (a similar proof works for $s$ odd). Since $\left(v^{s}\left(t^{0}, \ldots, v^{s-1}\right), \overline{v^{s-1}}\left(t^{1}, \ldots, t^{s-1}\right)\right) \in \mathcal{M}$, we see from (3-22) that

$$
\begin{aligned}
\partial^{\beta} H\left(v^{s}\left(t^{0}, \ldots, t^{s-1}\right)\right) \\
\quad \equiv \Psi_{\beta}^{v}\left(v^{s}\left(t^{0}, \ldots, t^{s-1}\right), \overline{v^{s-1}}\left(t^{1}, \ldots, t^{s-1}\right), j \frac{k+|\beta|}{v^{s-1}\left(t^{1}, \ldots, t^{s-1}\right)} \widetilde{H}\right) .
\end{aligned}
$$

Using (3-55), we see then that

$$
\begin{aligned}
\partial^{\beta} H\left(v^{s}\left(t^{0}, \ldots, t^{s-1}\right)\right) & \\
\equiv \Psi_{\beta}^{\nu}\left(v^{s}\left(t^{0}, \ldots, t^{s-1}\right), \overline{v^{s-1}}\left(t^{1}, \ldots, t^{s-1}\right),\right. & \left.\left(\overline{B_{s-1}^{v, \mu, \gamma}}\left(j_{0}^{k(s-1)+|\gamma|} H, t^{1}, \ldots, t^{s-1}\right)\right)_{|\gamma| \leq k+|\beta|}\right) .
\end{aligned}
$$

Now define $B_{s}^{\mu, \nu, \beta}\left(\Lambda, t^{0}, \ldots, t^{s-1}\right)$ to be the right hand side of (3-57), with the jets of $H$ replaced by the appropriate corresponding coordinates of $\Lambda$.

Using Lemma 3.5 , we see that $(\overline{\widetilde{H}}, \bar{H})$ satisfies condition $D_{v \mu}$ and sends $M$ into $\mathcal{M}^{\prime}$. So, we have from (3-54)

$$
\partial^{\gamma} \widetilde{H}\left(v^{s}\left(t^{0}, \ldots, t^{s-1}\right)\right)=B_{s}^{v, \mu, \gamma}\left(j_{0}^{k s+|\gamma|} \widetilde{H}, t^{0}, \ldots, t^{s-1}\right) .
$$

Taking the complex conjugate of both sides gives us (3-55).

Let $r$ be as given in Lemma 3.11. We know from (3-54) and (3-55) that

$$
\begin{aligned}
& H\left(v^{2 r}\left(t^{0}, \ldots, t^{2 r-1}\right)\right)=B_{2 r}^{\mu, v, 0}\left(j_{0}^{2 k r} H, t^{0}, \ldots, t^{2 r-1}\right), \\
& \widetilde{H}\left(\overline{v^{2 r+1}}\left(t^{0}, \ldots, t^{2 r}\right)\right)=\overline{B_{2 r+1}^{v, \mu, 0}}\left(j_{0}^{2 k r+k} H, t^{0}, \ldots, t^{2 r}\right) .
\end{aligned}
$$

Since $v^{l+1}\left(t^{0}, \ldots, t^{l-1}, 0\right)=v^{l}\left(t^{0}, \ldots, t^{l-1}\right)$ for any positive integer $l$, we see from Lemma 3.11 that the matrix

$$
\left(\frac{\partial v^{2 r+1}}{\partial\left(t^{0}, t^{r+1}, t^{r+2}, \ldots, t^{2 r-1}\right)}\left(0, x^{1}, \ldots, x^{r-1}, x^{r}, x^{r-1}, \ldots, x^{1}, 0\right)\right)
$$

has rank $m+d$ for all $\left(x_{1}, \ldots, x_{r}\right) \in U \backslash V$, for $U \subseteq \mathbb{C}^{r m}$ an open neighborhood of the origin and $V$ a proper holomorphic subvariety of $U$, and we also see that

$$
v^{2 r+1}\left(0, x^{1}, \ldots, x^{r-1}, x^{r}, x^{r-1}, \ldots, x^{1}, 0\right) \equiv 0 .
$$


We can now use (3-59) and (3-60) to obtain (3-52) and (3-53) by following exactly the proof of Theorem 3.3.

\section{Reformulation of Theorem 1.8.}

Theorem 3.15. Let $M$ and $M^{\prime}$ be as in Theorem 3.3, and assume that $M$ and $M^{\prime}$ are real algebraic. If $\mathscr{H}$ is a germ at 0 of an HSPM satisfying condition $D_{\mu \nu}$ for some $\mu$ and $\nu$, then $\mathscr{H}$ is holomorphic algebraic.

Proof. An inspection of the proof of Lemma 3.6 shows that the $\phi_{\beta}^{\alpha}$ as given in (3-10) are holomorphic algebraic (as $M$ is real algebraic). When solving the system of equations in (3-24), apply the algebraic implicit function theorem to see that $B^{v}$ as given in (3-25) is holomorphic algebraic (as $M^{\prime}$ is real algebraic). Thus, an inspection of the proof of Lemma 3.7 shows that the $\Psi_{\beta}^{\alpha}$ as given in (3-21) are holomorphic algebraic. An examination of the proof of Lemma 3.10 then reveals that the $\Xi_{s}^{\alpha, \beta}$ as given in (3-33) are holomorphic algebraic. Finally, in the proof of Theorem 3.3, choose $x_{0}$ sufficiently small and satisfying $\delta\left(x_{0}\right) \neq 0$, and substitute $x=x_{0}$ in (3-48). By Lemma 3.12, we see then that $H(Z)$ is holomorphic algebraic. Similarly, $\widetilde{H}(\zeta)$ is holomorphic algebraic.

\section{Proofs of main results}

In Section 1, we presented results 1.1, 1.2, 1.4, and 1.5, all of which follow naturally from Theorem 3.3. We also presented Theorem 1.6, which is a direct result of Theorem 3.14, and Theorem 1.8, which is a direct result of Theorem 3.15. In this section, we provide their proofs. First we make the following observations.

Observation 4.1. If $M \subseteq \mathbb{C}^{N}$ and $M^{\prime} \subseteq \mathbb{C}^{N^{\prime}}$ are generic submanifolds of codimensions $d$ and $d^{\prime}$, respectively, given in normal coordinates by $w=Q(z, \chi, \tau)$ and $w^{\prime}=Q^{\prime}\left(z^{\prime}, \chi^{\prime}, \tau^{\prime}\right)$, respectively, then a germ at 0 of an $\operatorname{HSPM} \mathscr{H}=(f, g, \tilde{f}, \tilde{g})$ sending $(\mathcal{M}, 0)$ into $\left(\mathcal{M}^{\prime}, 0\right)$ is Segre submersive at 0 if and only if the matrices $\left(f_{z}(0)\right)$ and $\left(\tilde{f}_{\chi}(0)\right)$ have rank $N^{\prime}-d^{\prime}$. This follows from the fact that a basis for the antiholomorphic vectors tangent to $M$ (respectively, $M^{\prime}$ ) at 0 is given by $\left\{\partial / \partial \bar{z}_{j}: 1 \leq j \leq N-d\right\}$ (respectively, $\left\{\partial / \partial \bar{z}_{j}^{\prime}: 1 \leq j \leq N^{\prime}-d^{\prime}\right\}$ ), while a basis for the holomorphic vectors tangent to $M$ (respectively, $M^{\prime}$ ) at 0 is given by $\left\{\partial / \partial z_{j}: 1 \leq j \leq N-d\right\}$ (respectively, $\left\{\partial / \partial z_{j}^{\prime}: 1 \leq j \leq N^{\prime}-d^{\prime}\right\}$ ), coupled with the fact that $g_{z_{j}}(0)=\tilde{g}_{\chi_{j}}(0)=0$ for $j=1, \ldots, N-d$.

Observation 4.2. For $p \in \mathbb{C}^{N}$, let $\phi:\left(\mathbb{C}^{N}, 0\right) \rightarrow\left(\mathbb{C}^{N}, p\right)$ be a biholomorphism near 0 , and for $p^{\prime} \in \mathbb{C}^{N^{\prime}}$, let $\phi^{\prime}:\left(\mathbb{C}^{N^{\prime}}, 0\right) \rightarrow\left(\mathbb{C}^{N^{\prime}}, p^{\prime}\right)$ be a biholomorphism near 0 . Then for any nonnegative $l$, there exist vector-valued polynomial functions $F_{l}$ and $G_{l}$ such that if $h:\left(\mathbb{C}^{N}, 0\right) \rightarrow\left(\mathbb{C}^{N^{\prime}}, 0\right)$ is any holomorphic map, and $\tilde{h}:\left(\mathbb{C}^{N}, p\right) \rightarrow$ $\left(\mathbb{C}^{N^{\prime}}, p^{\prime}\right)$ is given by $\tilde{h}:=\phi^{\prime} \circ h \circ \phi^{-1}$, then $j_{p}^{l} \tilde{h}=F_{l}\left(j_{0}^{l} h\right)$ and $j_{0}^{l} h=G_{l}\left(j_{p}^{l} \tilde{h}\right)$. 
Proof of Theorem 1.1. This theorem follows from Theorem 3.3, Observation 4.1, and Observation 4.2. The boundaries on $r$ given in Theorem 1.1 follow from the fact that there are $\left(\begin{array}{l}m \\ n\end{array}\right)$ possibilities for $\mu$ and $\left(\begin{array}{l}m \\ n\end{array}\right)$ possibilities for $v$ in Theorem 3.3. Therefore, there are $\left(\begin{array}{l}m \\ n\end{array}\right)\left(\begin{array}{l}m \\ n\end{array}\right)$ possible choices for $\Phi^{\alpha, \beta}$ in (3-3).

Proof of Corollary 1.2. Without loss of generality, assume $p=0$. Since $M=M^{\prime}$, we have $r=1$ in Theorem 1.1. Define $\Phi:=\Phi_{1}$ as given in (1-2). It then follows from Theorem 1.1 that $\eta_{0}^{K}$ is continuous and injective on $\operatorname{Aut}_{\mathbb{C}}(M, 0)$. To show that $\eta_{0}^{K}$ is a homeomorphism from $\operatorname{Aut}_{\mathbb{C}}(\mathcal{M}, 0)$ onto $\eta_{0}^{K}\left(\operatorname{Aut}_{\mathbb{C}}(M, 0)\right)$, we need to show the continuity of $\left(\eta_{0}^{K}\right)^{-1}$. Let $\Lambda_{j}, \widetilde{\Lambda}_{j}, \Lambda_{0}, \widetilde{\Lambda}_{0}$ belong to $G_{0}^{k}\left(\mathbb{C}^{N}\right)$ and assume $\left(\Lambda_{j}, \widetilde{\Lambda}_{j}\right) \in \eta_{0}^{K}\left(\operatorname{Aut}_{\mathbb{C}}(\mathcal{M}, 0)\right)$ converges to $\left(\Lambda_{0}, \widetilde{\Lambda}_{0}\right) \in \eta_{0}^{K}\left(\operatorname{Aut}_{\mathbb{C}}(\mathcal{M}, 0)\right)$. Then Theorem 1.1 tells us that

$$
\begin{aligned}
& \left(\eta_{0}^{K}\right)^{-1}\left(\Lambda_{j}, \widetilde{\Lambda}_{j}\right)=\left(\Phi\left(Z, \Lambda_{j}, \widetilde{\Lambda}_{j}\right), \bar{\Phi}\left(\zeta, \widetilde{\Lambda}_{j}, \Lambda_{j}\right)\right), \\
& \left(\eta_{0}^{K}\right)^{-1}\left(\Lambda_{0}, \widetilde{\Lambda}_{0}\right)=\left(\Phi\left(Z, \Lambda_{0}, \widetilde{\Lambda}_{0}\right), \bar{\Phi}\left(\zeta, \widetilde{\Lambda}_{0}, \Lambda_{0}\right)\right) .
\end{aligned}
$$

It follows that $\left(\eta_{0}^{K}\right)^{-1}\left(\Lambda_{j}, \widetilde{\Lambda}_{j}\right)$ converges to $\left(\eta_{0}^{K}\right)^{-1}\left(\Lambda_{0}, \widetilde{\Lambda}_{0}\right)$.

We now show $\eta_{0}^{K}\left(\operatorname{Aut}_{\mathbb{C}}(\mathcal{M}, 0)\right)$ is a closed, holomorphic algebraic submanifold of $G_{0}^{K}\left(\mathbb{C}^{N}\right) \times G_{0}^{K}\left(\mathbb{C}^{N}\right)$. Let $\rho(Z, \bar{Z})$ be a defining function for $M$ near 0 . Write $\zeta=\left(\zeta_{1}, \zeta_{2}\right) \in \mathbb{C}^{N-d} \times \mathbb{C}^{d}$, where $d$ is the codimension of $M$. After a possible rearrangement of coordinates, since $M$ is generic, there exists a holomorphic map $\theta: \mathbb{C}^{N} \times \mathbb{C}^{N-d} \rightarrow \mathbb{C}^{d}$ satisfying $\theta(0)=0$ such that for all $Z$ and $\zeta_{1}$ sufficiently close to 0 , we have $\left(Z, \zeta_{1}, \theta\left(Z, \zeta_{1}\right)\right) \in \mathcal{M}$. Given $\left(\Lambda_{0}, \widetilde{\Lambda}_{0}\right) \in G_{0}^{K}\left(\mathbb{C}^{N}\right) \times G_{0}^{K}\left(\mathbb{C}^{N}\right)$, $\left(\Lambda_{0}, \widetilde{\Lambda}_{0}\right) \in \eta_{0}^{K}\left(\operatorname{Aut}_{\mathbb{C}}(\mathcal{M}, 0)\right)$ if and only if the following three conditions hold:

$$
\begin{gathered}
\Lambda_{0}=\left(S_{\gamma}\left(\Lambda_{0}, \widetilde{\Lambda}_{0}\right)\right)_{|\gamma| \leq K}, \\
\widetilde{\Lambda}_{0}=\left(\overline{S_{\gamma}}\left(\widetilde{\Lambda}_{0}, \Lambda_{0}\right)\right)_{|\gamma| \leq K}, \\
\rho\left(\Phi\left(Z, \Lambda_{0}, \widetilde{\Lambda}_{0}\right), \bar{\Phi}\left(\zeta_{1}, \theta\left(Z, \zeta_{1}\right), \widetilde{\Lambda}_{0}, \Lambda_{0}\right)\right)=0,
\end{gathered}
$$

where $S_{\gamma}$ are the rational coefficients in the Taylor expansion given in (1-2). Equations (4-1) and (4-2) can be expressed as a finite set of polynomial equations in $\Lambda_{0}$ and $\widetilde{\Lambda}_{0}$ as each $S_{\gamma}$ is rational. We claim that (4-3) can be expressed as an infinite set of polynomial equations in $\Lambda_{0}$ and $\widetilde{\Lambda}_{0}$. Indeed, we can write the Taylor expansion as

$$
\rho\left(\Phi\left(Z, \Lambda_{0}, \widetilde{\Lambda}_{0}\right), \bar{\Phi}\left(\zeta_{1}, \theta\left(Z, \zeta_{1}\right), \widetilde{\Lambda}_{0}, \Lambda_{0}\right)\right)=\sum_{\alpha, \beta} R_{\alpha \beta}\left(\Lambda_{0}, \widetilde{\Lambda}_{0}\right) Z^{\alpha} \zeta_{1}^{\beta}
$$

Observe that each $R_{\alpha \beta}$ is rational. This can be seen by noting that $\Phi(0, \Gamma, \widetilde{\Lambda}) \equiv 0$ and $\theta(0)=0$, and by noting the form of $\Phi$ given in Theorem 1.1. The claim follows since the set of polynomial equations comes from setting the numerators of $R_{\alpha \beta}$ equal to 0 for all $\alpha$ and $\beta$. 
Thus, we see that $\eta_{0}^{K}\left(\operatorname{Aut}_{\mathbb{C}}(M, 0)\right)$ is a closed, holomorphic algebraic subvariety of the space $G_{0}^{K}\left(\mathbb{C}^{N}\right) \times G_{0}^{K}\left(\mathbb{C}^{N}\right)$ since it is given by the vanishing of a set of polynomial equations. To see it is actually a submanifold, note first that it is a subgroup of $G_{0}^{K}\left(\mathbb{C}^{N}\right) \times G_{0}^{K}\left(\mathbb{C}^{N}\right)$ since multiplication can be defined as follows. Given any $\left(\Lambda_{1}, \widetilde{\Lambda}_{1}\right),\left(\Lambda_{2}, \widetilde{\Lambda}_{2}\right) \in \eta_{0}^{K}\left(\operatorname{Aut}_{\mathbb{C}}(\mathcal{M}, 0)\right)$, let $\mathscr{H}_{1}$ and $\mathscr{H}_{2}$, respectively, be the corresponding automorphisms in $\operatorname{Aut}_{\mathbb{C}}(\mathcal{M}, 0)$. Now compose $\mathscr{H}_{1}$ and $\mathscr{H}_{2}$, and apply $\eta_{0}^{K}$ to this composition. Under this multiplication, $\eta_{0}^{K}\left(\operatorname{Aut}_{\mathbb{C}}(\mathcal{M}, 0)\right)$ is a closed subgroup of the Lie group $G_{0}^{K}\left(\mathbb{C}^{N}\right) \times G_{0}^{K}\left(\mathbb{C}^{N}\right)$, and is thus a Lie subgroup; see [Varadarajan 1974], for example.

\section{Proof of Corollary 1.4.}

Lemma 4.3. Let $A=\left(a_{i j}\right)$ be a $d \times d$ invertible matrix, where each $a_{i j} \in \mathbb{C}$. Let $b_{1}, \ldots, b_{d} \in \mathbb{C}$. Let $B_{1}$ be the matrix obtained by replacing row $m$ of $A$ with $\left(a_{m 1}+b_{1}, \ldots, a_{m d}+b_{d}\right)$ and $B_{2}$ be the matrix obtained by replacing row $m$ of $A$ with $\left(a_{m 1}-b_{1}, \ldots, a_{m d}-b_{d}\right)$. Then at least one of $B_{1}$ or $B_{2}$ is invertible.

Proof. Without loss of generality, assume $m=1$. Let $A_{n}:=(-1)^{n+1} \operatorname{det} M_{n}$, where $M_{n}$ is the $(d-1) \times(d-1)$ matrix obtained by deleting the first row and $n$-th column of $A$. Assume that det $B_{1}=\operatorname{det} B_{2}=0$. Then expanding along the first row of $B_{1}$ gives

$$
\left(a_{11}+b_{1}\right) A_{1}+\cdots+\left(a_{1 d}+b_{d}\right) A_{d}=0,
$$

and expanding along the first row of $B_{2}$ gives

$$
\left(a_{11}-b_{1}\right) A_{1}+\cdots+\left(a_{1 d}-b_{d}\right) A_{d}=0 .
$$

Adding (4-4) and (4-5) gives $2 a_{11} A_{1}+\ldots+2 a_{1 d} A_{d}=0$. However, this implies that $\operatorname{det} A=0$, a contradiction.

We now prove Corollary 1.4. Without loss of generality, assume $p=0$. Let $r(\Lambda, \bar{\Lambda})=\left(r_{1}(\Lambda, \bar{\Lambda}), \ldots, r_{s}(\Lambda, \bar{\Lambda})\right)$ be a defining function for $j_{0}^{K}(\operatorname{Aut}(M, 0))$ as a real algebraic submanifold of $G_{0}^{K}\left(\mathbb{C}^{N}\right)$, where $\Lambda \in G_{0}^{K}\left(\mathbb{C}^{N}\right)$; see Remark 1.3. The complexification $\mathbb{C}\left\{j_{0}^{K}(\operatorname{Aut}(M, 0))\right\}$ of this submanifold is thus a complex submanifold of $G_{0}^{K}\left(\mathbb{C}^{N}\right) \times G_{0}^{K}\left(\mathbb{C}^{N}\right)$ given by the vanishing of $r(\Lambda, \widetilde{\Lambda})$, where $\widetilde{\Lambda} \in G_{0}^{K}\left(\mathbb{C}^{N}\right)$. Let $\rho(Z, \bar{Z})$ be a defining function for $M$ near 0 . Since $M=M^{\prime}$, it is clear from the statement of Theorem 3.3 that we can choose $r=1$ in Theorem 1.1. Do so, and define $\Phi:=\Phi_{1}$ as given in (1-2). From Theorem 1.1, we see that for any $\Lambda \in G_{0}^{K}\left(\mathbb{C}^{N}\right)$,

$\rho(\Phi(Z, \Lambda, \bar{\Lambda}), \bar{\Phi}(\bar{Z}, \bar{\Lambda}, \Lambda))=A(Z, \Lambda, \bar{Z}, \bar{\Lambda}) r(\Lambda, \bar{\Lambda})+B(Z, \Lambda, \bar{Z}, \bar{\Lambda}) \rho(Z, \bar{Z})$, 
where $A$ is a real analytic $d \times s$ matrix, and $B$ is a real analytic $d \times d$ matrix. Complexify to get

$$
\rho(\Phi(Z, \Lambda, \widetilde{\Lambda}), \bar{\Phi}(\zeta, \widetilde{\Lambda}, \Lambda))=A(Z, \Lambda, \zeta, \widetilde{\Lambda}) r(\Lambda, \widetilde{\Lambda})+B(Z, \Lambda, \zeta, \widetilde{\Lambda}) \rho(Z, \zeta) .
$$

Notice that this gives us exactly what we want. This equation says that if $(\Lambda, \widetilde{\Lambda})$ is in $\mathbb{C}\left\{j_{0}^{K}(\operatorname{Aut}(M, 0))\right\}$, then $(\Phi(Z, \Lambda, \widetilde{\Lambda}), \bar{\Phi}(\zeta, \widetilde{\Lambda}, \Lambda))$ is in $\operatorname{Aut}_{\mathbb{C}}(\mathcal{M}, 0)$. Now we need only show that

$$
\eta_{0}^{K}(\Phi(Z, \Lambda, \widetilde{\Lambda}), \bar{\Phi}(\zeta, \widetilde{\Lambda}, \Lambda))=(\Lambda, \widetilde{\Lambda}) .
$$

We have the equations

$$
\begin{aligned}
& \left(\partial_{Z}^{\alpha} \Phi(0, \Lambda, \bar{\Lambda})\right)_{|\alpha| \leq K}=\Lambda+C(\Lambda, \bar{\Lambda}) r(\Lambda, \bar{\Lambda}), \\
& \left(\partial_{\bar{Z}}^{\alpha} \bar{\Phi}(0, \bar{\Lambda}, \Lambda)\right)_{|\alpha| \leq K}=\bar{\Lambda}+\bar{C}(\bar{\Lambda}, \Lambda) r(\Lambda, \bar{\Lambda}),
\end{aligned}
$$

for $C$ a real analytic matrix. Complexify these to get

$$
\begin{aligned}
& \left(\partial_{Z}^{\alpha} \Phi(0, \Lambda, \widetilde{\Lambda})\right)_{|\alpha| \leq K}=\Lambda+C(\Lambda, \widetilde{\Lambda}) r(\Lambda, \widetilde{\Lambda}), \\
& \left(\partial_{\zeta}^{\alpha} \bar{\Phi}(0, \widetilde{\Lambda}, \Lambda)\right)_{|\alpha| \leq K}=\widetilde{\Lambda}+\bar{C}(\widetilde{\Lambda}, \Lambda) r(\Lambda, \widetilde{\Lambda}),
\end{aligned}
$$

and the first part of Corollary 1.4 is proved.

Since we are assuming $p=0$, we take $\mathrm{Id}=\mathrm{Id}^{\prime}$ in Corollary 1.4. To prove the second part of Corollary 1.4, first we show that $\eta_{0}^{K}\left(\operatorname{Aut}_{\mathbb{C}}(M, 0)\right)$ is a complexified submanifold near (Id, Id). In other words, $\eta_{0}^{K}\left(\operatorname{Aut}_{\mathbb{C}}(\mathcal{M}, 0)\right)=\mathbb{C} R$, where $R$ is a real submanifold of $G_{0}^{K}\left(\mathbb{C}^{N}\right)$ (here $\mathbb{C} R$ denotes the complexification of $R$ ). We know from Corollary 1.2 that $\eta_{0}^{K}\left(\operatorname{Aut}_{\mathbb{C}}(M, 0)\right)$ is a complex submanifold of $G_{0}^{K}\left(\mathbb{C}^{N}\right) \times G_{0}^{K}\left(\mathbb{C}^{N}\right)$. Near $(\mathrm{Id}, \mathrm{Id})$, let $\hat{s}_{1}(\Lambda, \widetilde{\Lambda}), \ldots, \hat{s}_{t}(\Lambda, \widetilde{\Lambda})$ be defining functions for $\eta_{0}^{K}\left(\operatorname{Aut}_{\mathbb{C}}(\mathcal{M}, 0)\right)$. We will assume without loss of generality that these functions are defined on a ball $B$ of sufficiently small radius centered at (Id, Id); this way if $(\Gamma, \Lambda)$ is a point in $B$, then so is $(\Lambda, \Gamma)$ and $(\bar{\Lambda}, \bar{\Gamma})$. Now we set $s_{j}(\Lambda, \widetilde{\Lambda})$ to one of the following:

$$
\begin{aligned}
& s_{j}(\Lambda, \widetilde{\Lambda}):=\hat{s}_{j}(\Lambda, \widetilde{\Lambda})+\overline{\hat{s}}_{j}(\widetilde{\Lambda}, \Lambda), \\
& s_{j}(\Lambda, \widetilde{\Lambda}):=i \hat{s}_{j}(\Lambda, \widetilde{\Lambda})-i \overline{\hat{s}}_{j}(\widetilde{\Lambda}, \Lambda),
\end{aligned}
$$

We choose between these options as follows. Start with $j=1$. From Lemma 4.3, we can replace $\hat{s}_{1}$ with one of the above $s_{1}$, and in at least one case the differentials of $s_{1}, \hat{s}_{2}, \ldots, \hat{s}_{t}$ will be linearly independent near (Id, Id). Choose $s_{1}$ so that this is the case. Now do the same thing for $j=2$, then $j=3$, and so forth. Let $\mathscr{R}$ be the submanifold defined by $s_{1}(\Lambda, \widetilde{\Lambda})=\cdots=s_{t}(\Lambda, \widetilde{\Lambda})=0$. If $(\Lambda, \widetilde{\Lambda})$ is in $\eta_{0}^{K}\left(\operatorname{Aut}_{\mathbb{C}}(M, 0)\right)$, then from Lemma 3.5, we have $(\widetilde{\Lambda}, \bar{\Lambda}) \in \eta_{0}^{K}\left(\operatorname{Aut}_{\mathbb{C}}(M, 0)\right)$. Thus, $\hat{s}_{j}(\widetilde{\Lambda}, \bar{\Lambda})=0$, which implies $\overline{\hat{s}}_{j}(\widetilde{\Lambda}, \Lambda)=0$. In other words, near (Id, Id), 
$\eta_{0}^{K}\left(\operatorname{Aut}_{\mathbb{C}}(M, 0)\right) \subseteq \mathscr{R}$. But these two submanifolds have equal dimensions. So we see that, in fact, $\eta_{0}^{K}\left(\operatorname{Aut}_{\mathbb{C}}(\mathcal{M}, 0)\right)=\mathscr{R}$ near $(\mathrm{Id}, \mathrm{Id})$.

Now we need only show that $\mathscr{R}=\mathbb{C} R$ for some real submanifold $R \subseteq G_{0}^{K}\left(\mathbb{C}^{N}\right)$, and we will have proved our claim. Let

$$
R:=\left\{\Lambda: s_{1}(\Lambda, \bar{\Lambda})=\cdots=s_{t}(\Lambda, \bar{\Lambda})=0\right\} .
$$

Clearly $R$ is a nonempty set as it contains the point $\Lambda=$ Id. Since each $s_{j}$ is a real function and the differentials of $s_{1}, \ldots, s_{t}$ are linearly independent, $R$ is a real submanifold.

From Theorem 1.1, we see that if $(H, \widetilde{H}) \in \operatorname{Aut}_{\mathbb{C}}(M, 0)$ and $j_{0}^{K}(\widetilde{H})=j_{0}^{K}(\bar{H})$, we must have $\widetilde{H}=\bar{H}$. Thus, near (Id, Id),

$$
\begin{aligned}
\mathbb{C}\left\{j_{0}^{K}(\operatorname{Aut}(M, 0))\right\} \cap\{\widetilde{\Lambda}=\bar{\Lambda}\} & =\eta_{0}^{K}\left(\operatorname{Aut}_{\mathbb{C}}(\mathcal{M}, 0)\right) \cap\{\widetilde{\Lambda}=\bar{\Lambda}\} \\
& =\mathbb{C} R \cap\{\widetilde{\Lambda}=\bar{\Lambda}\},
\end{aligned}
$$

implying that $j_{0}^{K}(\operatorname{Aut}(M, 0))=R$. Thus their complexifications must be equal as well. That is, $\mathbb{C}\left\{j_{0}^{K}(\operatorname{Aut}(M, 0))\right\}=\eta_{0}^{K}\left(\operatorname{Aut}_{\mathbb{C}}(M, 0)\right)$ near $(\mathrm{Id}, \mathrm{Id})$. But both of these are algebraic holomorphic submanifolds. So if they are equal near (Id, Id), then using the notation given in the statement of this corollary, we must have $\mathscr{B}=\mathscr{C}$.

The third part of the corollary comes from the fact that $\eta_{0}^{K}\left(\operatorname{Aut}_{\mathbb{C}}(\mathcal{M}, 0)\right)$ is a Lie subgroup. Thus, each of its connected components is a coset of $\mathscr{B}$. Since $\mathbb{C}\left\{j_{0}^{K}(\operatorname{Aut}(M, 0))\right\} \subseteq \eta_{0}^{K}\left(\operatorname{Aut}_{\mathbb{C}}(\mathcal{M}, 0)\right)$ and they are both algebraic holomorphic submanifolds, each component of $\mathbb{C}\left\{j_{0}^{K}(\operatorname{Aut}(M, 0))\right\}$ is exactly equal to one of the components of $\eta_{0}^{K}\left(\operatorname{Aut}_{\mathbb{C}}(\mathcal{M}, 0)\right)$. Algebraicity implies that there are finitely many such components.

Proof of Theorem 1.5. Assume first that $M$ and $M^{\prime}$ are given in normal coordinates and that $p=0$ and $p^{\prime}=0$. The equalities of the $K$-jets imply, in particular, that $\mathscr{H}_{1}$ and $\mathscr{H}_{2}$ both satisfy condition $D_{\mu \nu}$ for some $\mu$ and $\nu$. Theorem 1.5 now follows from Theorem 3.3 and Observations 4.1 and 4.2.

Proof of Theorem 1.6. This follows from Theorem 3.14 and Observations 4.1 and 4.2. We leave the details to the reader.

Proof of Theorem 1.8. As $M$ and $M^{\prime}$ are real algebraic, they have real analytic algebraic defining functions. When $M$ and $M^{\prime}$ are expressed in normal coordinates, the new defining functions can also be chosen to be real analytic algebraic. This follows by using the algebraic implicit function theorem in the derivation of the new defining functions; see [Baouendi et al. 1999b] for precise details on deriving normal coordinates and the algebraic implicit function theorem. Also, if $\widetilde{Z}=\varphi(Z)$ is a holomorphic algebraic change of coordinates, then $\varphi^{-1}$ is a holomorphic algebraic function; this is also a direct consequence of the algebraic implicit function theorem. Theorem 1.8 now follows from Theorem 3.15 and Observation 4.1. 


\section{Examples: HSPMs and automorphism groups}

For $n>1$, there exist $M, M^{\prime} \subseteq \mathbb{C}^{n+1}$ defined near 0 such that there exist no holomorphic maps $H$ satisfying

$$
H \text { is invertible near } 0, \quad H(M) \subseteq M^{\prime}, \quad H(0)=0,
$$

yet there exist HSPMs satisfying

$$
\mathscr{H} \text { is invertible near } 0, \quad \mathscr{H}(\mathcal{M}) \subseteq \mathcal{M}^{\prime}, \quad \mathscr{H}(0)=0 .
$$

Example 5.1. For $n>1$, let $\left(z_{1}, \ldots, z_{n}, w\right)$ and $\left(z_{1}^{\prime}, \ldots, z_{n}^{\prime}, w^{\prime}\right)$ be coordinates on $\mathbb{C}^{n+1}$ and define

$$
M=\left\{\operatorname{Im} w=\sum_{j=1}^{n} \epsilon_{j}\left|z_{j}\right|^{2}\right\} \quad \text { and } \quad M^{\prime}=\left\{\operatorname{Im} w^{\prime}=\sum_{j=1}^{n} \sigma_{j}\left|z_{j}^{\prime}\right|^{2}\right\},
$$

where $\epsilon_{j}, \sigma_{j} \in\{-1,1\}$. Both $M$ and $M^{\prime}$ are of finite type and finitely nondegenerate at 0 . If $\left|\sum_{j} \epsilon_{j}\right| \neq\left|\sum_{j} \sigma_{j}\right|$, then there are no holomorphic maps satisfying criteria (5-1). (Indeed, $M$ and $M^{\prime}$ have different Levi signatures at 0 .) However, for $a, c_{j} \in \mathbb{C} \backslash\{0\}$, the family of maps given by

$$
\begin{aligned}
& \mathscr{H}(z, w, \chi, \tau) \\
& =\left(\epsilon_{1} c_{1} z_{1}, \ldots, \epsilon_{n-1} c_{n-1} z_{n-1}, \epsilon_{n} c_{n} z_{n}, a w, \frac{a \sigma_{1}}{c_{1}} \chi_{1}, \ldots, \frac{a \sigma_{n-1}}{c_{n-1}} \chi_{n-1}, \frac{a \sigma_{n}}{c_{n}} \chi_{n}, a \tau\right)
\end{aligned}
$$

satisfy criteria (5-2).

This can also occur in $\mathbb{C}^{2}$ as the next example illustrates.

Example 5.2. Let $M, M^{\prime} \subseteq \mathbb{C}^{2}$ be given by

$$
\begin{aligned}
M & =\left\{\operatorname{Im} w=|z|^{2}+2 \operatorname{Re}\left(z^{4} \bar{z}^{2}(1+i \operatorname{Re} w)\right)\right\}, \\
M^{\prime} & =\left\{\operatorname{Im} w^{\prime}=\left|z^{\prime}\right|^{2}+2 \operatorname{Re}\left(z^{\prime 4} \bar{z}^{\prime 2}\left(1-i \operatorname{Re} w^{\prime}\right)\right)\right\} .
\end{aligned}
$$

Notice that $M$ and $M^{\prime}$ are of finite type and finitely nondegenerate at 0 . It can be shown [Chern and Moser 1974] that there are no maps $H$ satisfying criteria (5-1). (Indeed, as $M$ and $M^{\prime}$ are in Chern-Moser normal form, the fact that the coefficients $i$ and $-i$ are unequal implies that there does not exist a holomorphic map $H$ satisfying criteria (5-1).) However, it is easy to check that the HSPM $\mathscr{H}(z, w, \chi, \tau)=(i z,-w, i \chi,-\tau)$ satisfies (5-2).

Now we will look at some examples of automorphism groups. In Example 5.3, we find that $\mathbb{C}\left\{j_{0}^{K}(\operatorname{Aut}(M, 0))\right\}$ and $\eta_{0}^{K}\left(\operatorname{Aut}_{\mathbb{C}}(\mathcal{M}, 0)\right)$ are equal.

Example 5.3. Let $M$ be the Lewy hypersurface of $\mathbb{C}^{2}$. It is given by

$$
M=\left\{\operatorname{Im} w=|z|^{2}\right\} .
$$


We note that $M$ is finitely nondegenerate and of finite type at 0 . It can be shown (see [Angle 2008b] for the calculations) that every holomorphic Segre-preserving automorphism of $\mathcal{M}$ at 0 is of the form

$$
\begin{aligned}
\mathscr{H}(z, w, \chi, \tau)= & \frac{\alpha(z+\beta w)}{1-(\gamma+i \beta \tilde{\beta}) w-2 i \tilde{\beta} z}, \frac{\alpha \tilde{\alpha} w}{1-(\gamma+i \beta \tilde{\beta}) w-2 i \tilde{\beta} z}, \\
\left.\frac{\tilde{\alpha}(\chi+\tilde{\beta} \tau)}{1-(\gamma-i \beta \tilde{\beta}) \tau+2 i \beta \chi}, \frac{\alpha \tilde{\alpha} \tau}{1-(\gamma-i \beta \tilde{\beta}) \tau+2 i \beta \chi}\right) & ,
\end{aligned}
$$

where $\gamma, \beta, \tilde{\beta} \in \mathbb{C}$ and $\alpha, \tilde{\alpha} \in \mathbb{C} \backslash\{0\}$. Also, every automorphism of $M$ at 0 is of the form

$$
H(z, w)=\left(\frac{\alpha(z+\beta w)}{1-\left(\gamma+i|\beta|^{2}\right) w-2 i \bar{\beta} z}, \frac{|\alpha|^{2} w}{1-\left(\gamma+i|\beta|^{2}\right) w-2 i \bar{\beta} z}\right),
$$

where $\alpha \in \mathbb{C} \backslash\{0\}, \beta \in \mathbb{C}$, and $\gamma \in \mathbb{R}$. The automorphisms in (5-4) follow directly from the automorphisms in (5-3), but those in (5-4) have actually been known for some time [Chern and Moser 1974].

We use (5-3) and (5-4) to show that $\mathbb{C}\left\{j_{0}^{K}(\operatorname{Aut}(M, 0))\right\}=\eta_{0}^{K}\left(\operatorname{Aut}_{\mathbb{C}}(M, 0)\right)$ : Let

$$
\left(\Lambda_{z}^{f}, \ldots, \Lambda_{w w}^{f}, \Lambda_{z}^{g}, \ldots, \Lambda_{w w}^{g}, \Lambda_{\chi}^{\tilde{f}}, \ldots, \Lambda_{\tau \tau}^{\tilde{f}}, \Lambda_{\chi}^{\tilde{g}}, \ldots, \Lambda_{\tau \tau}^{\tilde{g}}\right)
$$

be coordinates on $G_{0}^{2}\left(\mathbb{C}^{2}\right) \times G_{0}^{2}\left(\mathbb{C}^{2}\right)$, where

$$
\begin{array}{ll}
\Lambda_{z^{r} w^{s}}^{f} \text { corresponds to } \frac{\partial^{r+s} f}{\partial z^{r} \partial w^{s}}, & \Lambda_{z^{r} w^{s}}^{g} \text { corresponds to } \frac{\partial^{r+s} g}{\partial z^{r} \partial w^{s}}, \\
\Lambda_{\chi^{r} \tau^{s}}^{\tilde{f}} \text { corresponds to } \frac{\partial^{r+s} \tilde{f}}{\partial \chi^{r} \partial \tau^{s}}, & \Lambda_{\chi^{r} \tau^{s}}^{\tilde{g}} \text { corresponds to } \frac{\partial^{r+s} \tilde{g}}{\partial \chi^{r} \partial \tau^{s}} .
\end{array}
$$

Then (5-4) implies that $\mathbb{C}\left\{j_{0}^{2}(\operatorname{Aut}(M, 0))\right\}$ is given by

$$
\begin{aligned}
& \left\{\Lambda_{w}^{g}=\Lambda_{\tau}^{\tilde{g}}=\Lambda_{z}^{f} \Lambda_{\chi}^{\tilde{f}}, \quad \Lambda_{w w}^{g}=\Lambda_{\tau \tau}^{\tilde{g}}+2 i \Lambda_{w}^{f} \Lambda_{\tau}^{\tilde{f}},\right. \\
& \Lambda_{z w}^{g}=2 i \Lambda_{z}^{f} \Lambda_{\tau}^{\tilde{f}}, \quad \Lambda_{\chi \tau}^{\tilde{g}}=-2 i \Lambda_{\chi}^{\tilde{f}} \Lambda_{w}^{f}, \\
& \Lambda_{z w}^{f}=\frac{\Lambda_{w w}^{g}}{\Lambda_{\chi}^{\tilde{f}}}, \quad \Lambda_{z z}^{f}=2 i \frac{\Lambda_{z}^{f} \Lambda_{\tau}^{\tilde{f}}}{\Lambda_{\chi}^{\tilde{f}}}, \quad \Lambda_{w w}^{f}=\frac{\Lambda_{w w}^{g} \Lambda_{w}^{f}}{\Lambda_{z}^{f} \Lambda_{\chi}^{\tilde{f}}}, \\
& \Lambda_{\chi \tau}^{\tilde{f}}=\frac{\Lambda_{\tau \tau}^{\tilde{g}}}{\Lambda_{z}^{f}}, \quad \Lambda_{\chi \chi}^{\tilde{f}}=-2 i \frac{\Lambda_{\chi}^{\tilde{f}} \Lambda_{w}^{f}}{\Lambda_{z}^{f}}, \quad \Lambda_{\tau \tau}^{\tilde{f}}=\frac{\Lambda_{\tau \tau}^{\tilde{g}} \Lambda_{\tau}^{\tilde{f}}}{\Lambda_{\chi}^{\tilde{f}} \Lambda_{z}^{f}}, \\
& \left.\Lambda_{z}^{g}=\Lambda_{z z}^{g}=\Lambda_{\chi}^{\tilde{g}}=\Lambda_{\chi \chi}^{\tilde{g}}=0\right\} .
\end{aligned}
$$

It follows from (5-3) that $\eta_{0}^{2}\left(\operatorname{Aut}_{\mathbb{C}}(\mathcal{M}, 0)\right)$ is also given by (5-5). 
More interesting, however, are submanifolds for which $\mathbb{C}\left\{j_{0}^{K}(\operatorname{Aut}(M, 0))\right\}$ is not equal to $\eta_{0}^{K}\left(\operatorname{Aut}_{\mathbb{C}}(M, 0)\right)$.

Example 5.4. Let $M \subseteq \mathbb{C}^{2}$ be given by

$$
M=\left\{\operatorname{Im} w=|z|^{2}+\left(\operatorname{Re} z^{2}\right)|z|^{2}\right\} .
$$

Note that $M$ is finitely nondegenerate and of finite type at 0 . Baouendi et al. [1997] showed that there are only two automorphisms of $M$ at 0 , namely $H_{1}(z, w)=(z, w)$ and $H_{2}(z, w)=(-z, w)$. Thus, $\mathbb{C}\left\{j_{0}^{K}(\operatorname{Aut}(M, 0))\right\}$ also has only two elements. However, the group of holomorphic Segre-preserving automorphisms of $M$ at 0 (which according to Corollary 1.4 consists of a finite number of elements) contains at least four maps,

$$
\begin{array}{ll}
\mathscr{H}_{1}(z, w, \chi, \tau)=(z, w, \chi, \tau), & \mathscr{H}_{2}(z, w, \chi, \tau)=(-z, w,-\chi, \tau), \\
\mathscr{H}_{3}(z, w, \chi, \tau)=(-z,-w, \chi,-\tau), & \mathscr{H}_{4}(z, w, \chi, \tau)=(z,-w,-\chi,-\tau) .
\end{array}
$$

The next two examples will compare $\eta_{0}^{K}\left(\operatorname{Aut}_{\mathbb{C}}(\mathcal{M}, 0)\right)$ and $\mathbb{C}\left\{j_{0}^{K}(\operatorname{Aut}(M, 0))\right\}$ for the family $\mathscr{F}$ given by

$$
\mathscr{F}_{\mathcal{F}}=\left\{M=\left.\left\{\operatorname{Im} w=c_{1}|z|^{2 m}+c_{2}|z|^{2 n}\right\}|1<m<n,| c_{1}\right|^{2}+\left|c_{2}\right|^{2} \neq 0\right\} .
$$

We exclude the Levi flat case, $M=\{\operatorname{Im} w=0\}$, since there is no finite jet determination for this $M$. Notice that each submanifold in $\mathscr{F}$ is of finite type and finitely degenerate at 0.

Example 5.5. Assume $c_{1} \neq 0$ and $c_{2}=0$. Calculations in [Angle 2008b] show that any holomorphic Segre-preserving automorphism of $\mathcal{M}$ at 0 is given by

$$
\begin{aligned}
\mathscr{H}(z, w, \chi, \tau) & =(f(z, w), g(z, w), \tilde{f}(\chi, \tau), \tilde{g}(\chi, \tau)) \\
& =\left(\frac{a z}{\sqrt[m]{1+\alpha w}}, \frac{a^{m} \tilde{a}^{m} w}{1+\alpha w}, \frac{\tilde{a} \chi}{\sqrt[m]{1+\alpha \tau}}, \frac{a^{m} \tilde{a}^{m} \tau}{1+\alpha \tau}\right),
\end{aligned}
$$

where $a, \tilde{a} \in \mathbb{C} \backslash\{0\}, \alpha \in \mathbb{C}$, and $f$ and $\tilde{f}$ are expressed in terms of any branch of the $m$-th root.

It immediately follows that any automorphism of $M$ at 0 is of the form

$$
H(z, w)=\left(\frac{a z}{\sqrt[m]{1+\alpha w}}, \frac{|a|^{2 m} w}{1+\alpha w}\right)
$$

where $a \in \mathbb{C} \backslash\{0\}, \alpha \in \mathbb{R}$, and $f$ is expressed in terms of any branch of the $m$-th root. 
In this case, $\eta_{0}^{2}\left(\operatorname{Aut}_{\mathbb{C}}(M, 0)\right)=\mathbb{C}\left\{j_{0}^{2}(\operatorname{Aut}(M, 0))\right\}$. Indeed, from (5-7), we see that $\mathbb{C}\left\{j_{0}^{2}(\operatorname{Aut}(M, 0))\right\}$ is given by

$$
\begin{aligned}
\left\{\Lambda_{w}^{g}=\Lambda_{\tau}^{\tilde{g}}\right. & =\left(\Lambda_{z}^{f}\right)^{m}\left(\Lambda_{\chi}^{\tilde{f}}\right)^{m}, \quad \Lambda_{w w}^{g}=2 m\left(\Lambda_{z}^{f}\right)^{m-1}\left(\Lambda_{\chi}^{\tilde{f}}\right)^{m} \Lambda_{z w}^{f}, \\
\Lambda_{\tau \tau}^{\tilde{g}} & =2 m\left(\Lambda_{\chi}^{\tilde{f}}\right)^{m-1}\left(\Lambda_{z}^{f}\right)^{m} \Lambda_{\chi \tau}^{\tilde{f}}, \\
\Lambda_{w}^{f} & =\Lambda_{w w}^{f}=\Lambda_{z z}^{f}=\Lambda_{z}^{g}=\Lambda_{z w}^{g}=\Lambda_{z z}^{g} \\
& \left.=\Lambda_{\tau}^{\tilde{f}}=\Lambda_{\tau \tau}^{\tilde{f}}=\Lambda_{\chi \chi}^{\tilde{f}}=\Lambda_{\chi}^{\tilde{g}}=\Lambda_{\chi \tau}^{\tilde{g}}=\Lambda_{\chi \chi}^{\tilde{g}}=0\right\} .
\end{aligned}
$$

It follows from (5-6) that $\eta_{0}^{2}\left(\operatorname{Aut}_{\mathbb{C}}(\mathcal{M}, 0)\right)$ is also given by (5-8).

Example 5.6. Assume $c_{1}, c_{2} \neq 0$. Calculations in [Angle 2008b] show that any holomorphic Segre-preserving automorphism of $M$ at 0 is of one of the $n-m$ forms

$$
\mathscr{H}_{c}(z, w, \chi, \tau)=\left(a z, c^{m} w,(c / a) \chi, c^{m} \tau\right),
$$

where $a \in \mathbb{C} \backslash\{0\}$ and $c \in\left\{e^{2 i \pi r /(n-m)}: r=0, \ldots, n-m-1\right\}$ (that is, $c$ is a primitive $(n-m)$-th root of unity).

It immediately follows that any automorphism of $M$ at 0 is of the form

$$
H(z, w)=\left(e^{i \theta} z, w\right), \quad \text { where } \theta \in \mathbb{R} .
$$

Thus, we see from (5-10) that $\mathbb{C}\left\{j_{0}^{1}(\operatorname{Aut}(M, 0))\right\}$ is given by

$$
\left\{\Lambda_{w}^{g}=\Lambda_{\tau}^{\tilde{g}}=1, \Lambda_{z}^{f} \Lambda_{\chi}^{\tilde{f}}=1, \Lambda_{w}^{f}=\Lambda_{\tau}^{\tilde{f}}=\Lambda_{z}^{g}=\Lambda_{\chi}^{\tilde{g}}=0\right\}
$$

and thus has positive dimension. For $n=m+1,(5-9)$ implies $\mathbb{C}\left\{j_{0}^{1}(\operatorname{Aut}(M, 0))\right\}=$ $\eta_{0}^{1}\left(\operatorname{Aut}_{\mathbb{C}}(M, 0)\right)$. For $n>m+1$, however, $\mathbb{C}\left\{j_{0}^{1}(\operatorname{Aut}(M, 0))\right\} \varsubsetneqq \eta_{0}^{1}\left(\operatorname{Aut}_{\mathbb{C}}(M, 0)\right)$. Indeed, we see from (5-9) that $\eta_{0}^{1}\left(\operatorname{Aut}_{\mathbb{C}}(\mathcal{M}, 0)\right)$ is equal to the disjoint union of exactly $n-m$ distinct cosets of $\mathbb{C}\left\{j_{0}^{1}(\operatorname{Aut}(M, 0))\right\}$.

\section{References}

[Angle 2008a] R. B. Angle, "Geometric properties and related results for holomorphic Segre preserving maps", preprint, 2008. To appear in Complex Var. Elliptic Equ. arXiv 0810.2570

[Angle 2008b] R. B. Angle, Holomorphic Segre preserving maps, thesis, University of California, San Diego, 2008, available at http://tinyurl.com/3ybdqjb. MR 2626910

[Baouendi et al. 1996] M. S. Baouendi, P. Ebenfelt, and L. P. Rothschild, "Algebraicity of holomorphic mappings between real algebraic sets in $\mathbf{C}^{n}$ ", Acta Math. 177 (1996), 225-273. MR 99b:32030 Zbl 0890.32005

[Baouendi et al. 1997] M. S. Baouendi, P. Ebenfelt, and L. P. Rothschild, "Parametrization of local biholomorphisms of real analytic hypersurfaces", Asian J. Math. 1:1 (1997), 1-16. MR 99b:32022 Zbl 0943.32021

[Baouendi et al. 1999a] M. S. Baouendi, P. Ebenfelt, and L. P. Rothschild, "Rational dependence of smooth and analytic CR mappings on their jets", Math. Ann. 315:2 (1999), 205-249. MR 2001b: 32075 Zbl 0942.32027 
[Baouendi et al. 1999b] M. S. Baouendi, P. Ebenfelt, and L. P. Rothschild, Real submanifolds in complex space and their mappings, Princeton Math. Series 47, Princeton University Press, 1999. MR 2000b:32066 Zbl 0944.32040

[Baouendi et al. 2001] M. S. Baouendi, L. P. Rothschild, and D. Zaitsev, "Equivalences of real submanifolds in complex space", J. Differential Geom. 59:2 (2001), 301-351. MR 2003k:32051 Zbl 1037.32030

[Bloom and Graham 1977] T. Bloom and I. Graham, "On "type" conditions for generic real submanifolds of $\mathbf{C}^{n}$ ”, Invent. Math. 40:3 (1977), 217-243. MR 58 \#28644 Zbl 0346.32013

[Chern 1975] S. S. Chern, "On the projective structure of a real hypersurface in $C_{n+1}$ ", Math. Scand. 36 (1975), 74-82. MR 52 \#814 Zbl 0305.53019

[Chern and Moser 1974] S. S. Chern and J. K. Moser, "Real hypersurfaces in complex manifolds", Acta Math. 133 (1974), 219-271. MR 54 \#13112 Zbl 0302.32015

[Faran 1980] J. J. Faran, "Segre families and real hypersurfaces", Invent. Math. 60:2 (1980), 135172. MR 81i:32018 Zbl 0464.32011

[Kohn 1972] J. J. Kohn, "Boundary behavior of $\delta$ on weakly pseudo-convex manifolds of dimension two", J. Differential Geometry 6 (1972), 523-542. MR 48 \#727 Zbl 0256.35060

[Lamel and Mir 2007] B. Lamel and N. Mir, "Parametrization of local CR automorphisms by finite jets and applications", J. Amer. Math. Soc. 20 (2007), 519-572. MR 2008b:32025 Zbl 1112.32017

[Segre 1931] B. Segre, "Intorno al problema di Poincaré della rappresentazione pseudoconforme", Rendiconti Accad. d. L. Roma 13 (1931), 676-683. JFM 57.0404.05

[Varadarajan 1974] V. S. Varadarajan, Lie groups, Lie algebras, and their representations, PrenticeHall, Englewood Cliffs, N.J., 1974. MR 51 \#13113 Zbl 0371.22001

[Zhang 2007] Y. Zhang, "Rigidity and holomorphic Segre transversality for holomorphic Segre maps”, Math. Ann. 337:2 (2007), 457-478. MR 2008e:32021 Zbl 1117.32012

Received May 21, 2009.

R. BLAir ANGLE

DEPARTMENT OF MATHEMATICS

UNIVERSITY OF CALIFORNIA AT SAN DIEGo

LA JOLLA, CA 92093-0112

UNITED STATES

angle@metsci.com 


\title{
PACIFIC JOURNAL OF MATHEMATICS
}

\author{
http://www.pjmath.org \\ Founded in 1951 by \\ E. F. Beckenbach (1906-1982) and F. Wolf (1904-1989)
}

\section{EDITORS}

V. S. Varadarajan (Managing Editor)

Department of Mathematics

University of California

Los Angeles, CA 90095-1555

pacific@math.ucla.edu

Vyjayanthi Chari

Department of Mathematics

University of California

Riverside, CA 92521-0135

chari@math.ucr.edu

Robert Finn

Department of Mathematics Stanford University

Stanford, CA 94305-2125

finn@math.stanford.edu

Kefeng Liu

Department of Mathematics

University of California

Los Angeles, CA 90095-1555

liu@math.ucla.edu
Darren Long

Department of Mathematics

University of California

Santa Barbara, CA 93106-3080

long@math.ucsb.edu

Jiang-Hua Lu

Department of Mathematics

The University of Hong Kong

Pokfulam Rd., Hong Kong jhlu@maths.hku.hk

Alexander Merkurjev

Department of Mathematics

University of California

Los Angeles, CA 90095-1555

merkurev@math.ucla.edu
Sorin Popa

Department of Mathematics University of California

Los Angeles, CA 90095-1555 popa@math.ucla.edu

Jie Qing

Department of Mathematics

University of California

Santa Cruz, CA 95064

qing@cats.ucsc.edu

Jonathan Rogawski

Department of Mathematics

University of California

Los Angeles, CA 90095-1555

jonr@math.ucla.edu

\section{PRODUCTION}

pacific@math.berkeley.edu

\begin{abstract}
Silvio Levy, Scientific Editor Matthew Cargo, Senior Production Editor
\end{abstract}
ACADEMIA SINICA, TAIPEI

CALIFORNIA INST. OF TECHNOLOGY

INST. DE MATEMÁTICA PURA E APLICADA

KEIO UNIVERSITY

MATH. SCIENCES RESEARCH INSTITUTE

NEW MEXICO STATE UNIV.

OREGON STATE UNIV.

\section{SUPPORTING INSTITUTIONS}

STANFORD UNIVERSITY
UNIV. OF BRITISH COLUMBIA
UNIV. OF CALIFORNIA, BERKELEY
UNIV. OF CALIFORNIA, DAVIS
UNIV. OF CALIFORNIA, LOS ANGELES
UNIV. OF CALIFORNIA, RIVERSIDE
UNIV. OF CALIFORNIA, SAN DIEGO
UNIV. OF CALIF., SANTA BARBARA

UNIV. OF CALIF., SANTA CRUZ

UNIV. OF MONTANA

UNIV. OF OREGON

UNIV. OF SOUTHERN CALIFORNIA

UNIV. OF UTAH

UNIV. OF WASHINGTON

WASHINGTON STATE UNIVERSITY

These supporting institutions contribute to the cost of publication of this Journal, but they are not owners or publishers and have no responsibility for its contents or policies.

See inside back cover or www.pjmath.org for submission instructions.

The subscription price for 2010 is US \$420/year for the electronic version, and \$485/year for print and electronic.

Subscriptions, requests for back issues from the last three years and changes of subscribers address should be sent to Pacific Journal of Mathematics, P.O. Box 4163, Berkeley, CA 94704-0163, U.S.A. Prior back issues are obtainable from Periodicals Service Company, 11 Main Street, Germantown, NY 12526-5635. The Pacific Journal of Mathematics is indexed by Mathematical Reviews, Zentralblatt MATH, PASCAL CNRS Index, Referativnyi Zhurnal, Current Mathematical Publications and the Science Citation Index.

The Pacific Journal of Mathematics (ISSN 0030-8730) at the University of California, c/o Department of Mathematics, 969 Evans Hall, Berkeley, CA 94720-3840, is published monthly except July and August. Periodical rate postage paid at Berkeley, CA 94704, and additional mailing offices. POSTMASTER: send address changes to Pacific Journal of Mathematics, P.O. Box 4163, Berkeley, CA 94704-0163.

PJM peer review and production are managed by EditFLOW ${ }^{\mathrm{TM}}$ from Mathematical Sciences Publishers.

PUBLISHED BY PACIFIC JOURNAL OF MATHEMATICS

at the University of California, Berkeley 94720-3840

A NON-PROFIT CORPORATION

Typeset in LATEX

Copyright $(\mathrm{C} 2010$ by Pacific Journal of Mathematics 


\section{PACIFIC JOURNAL OF MATHEMATICS}

Volume $247 \quad$ No. $2 \quad$ October 2010

A family of representations of braid groups on surfaces

257

ByUnG HeE AN and Ki HyOUNG Ko

Parametrization of holomorphic Segre-preserving maps

283

R. BLAIR ANGLE

Chern classes on differential $K$-theory

ULRICH BUNKE

Laplacian spectrum for the nilpotent Kac-Moody Lie algebras

DMITRY FUCHS and CONSTANCE WILMARTH

Sigma theory and twisted conjugacy classes

DACIBERG GONÇALVES and DESSISLAVA Hristova Kochloukova

Properties of annular capillary surfaces with equal contact angles

JAMES GORDON and DAVID SIEGEL

Approximating annular capillary surfaces with equal contact angles

JAMES GORDON and DAVID SIEGEL

Harmonic quasiconformal self-mappings and Möbius transformations of the unit ball

David Kalaj and Miodrag S. Mateljević

Klein bottle and toroidal Dehn fillings at distance 5

SANGYOP LEE

Representations of the two-fold central extension of $\mathrm{SL}_{2}\left(\mathbb{Q}_{2}\right)$

HUNG YEAN LOKE and GORDAN SAVIN

Large quantum corrections in mirror symmetry for a 2-dimensional

Lagrangian submanifold with an elliptic umbilic

Giovanni MARELLI

Crossed pointed categories and their equivariantizations

DEEPAK NAIDU

Painlevé analysis of generalized Zakharov equations

Hassan A. ZeDan and Salma M. Al-Tuwairqi 